\title{
Recovery of Mercury from Contaminated Liquid Wastes
}

\author{
Topical Report \\ Covering Base Contract \\ November 27, 1996 through June 24, 1998 \\ Contract Number DE-RA21-97MC32195--15 \\ ADA Project Number 4446
}

\author{
prepared for \\ Federal Energy Technology Center - Morgantown \\ P. O. Box 880 \\ Morgantown, WV 26507-0880
}

prepared by

ADA Technologies, Inc.

304 Inverness Way South, Suite 365

Englewood, CO 80112

June 12, 1998 


\section{Disclaimer}

This report was prepared as an account of work sponsored by an agency of the United States Government. Neither the United States Government nor any agency thereof, nor any of their employees, makes any warranty, express or implied, or assumes any legal liability or responsibility for the accuracy, completeness, or usefulness of any information, apparatus, product, or process disclosed, or represents that its use would not infringe privately owned rights. Reference herein to any specific commercial product, process, or service by trade

name, trademark, manufacturer, or otherwise does not necessarily constitute or imply its endorsement, recommendation, or favoring by the United States Government or any agency thereof. The views and opinions of authors expressed herein do not necessarily state or reflect those of the United States Government or any agency thereof. 


\begin{abstract}
The Base Contract program emphasized the manufacture and testing of superior sorbents for mercury removal, testing of the sorption process at a DOE site, and determination of the regeneration conditions in the laboratory. During this project, ADA Technologies, Inc. demonstrated the following key elements of a successful regenerable mercury sorption process: 1) sorbents that have a high capacity for dissolved, ionic mercury; 2) removal of ionic mercury at greater than $99 \%$ efficiency; and 3) thermal regeneration of the spent sorbent.

ADA's process is based on the highly efficient and selective sorption of mercury by noble metals. Contaminated liquid flows through two packed columns that contain microporous sorbent particles on which a noble metal has been finely dispersed. A third column is held in reserve. When the sorbent is loaded with mercury to the point of breakthrough at the outlet of the second column, the first column is taken off-line and the flow of contaminated liquid is switched to the second and third columns. The spent column is regenerated by heating. A small flow of purge gas carries the desorbed mercury to a capture unit where the liquid merucry is recovered.

Laboratory-scale tests with mercuric chloride solutions demonstrated the sorbents' ability to remove mercury from contaminated wastewater. Isotherms on surrogate wastes from DOE's Y12 Plant in Oak Ridge, Tennessee showed greater than $99.9 \%$ mercury removal. Laboratory- and pilot-scale tests on actual Y-12 Plant wastes were also successful. Mercury concentrations were reduced to less than $1 \mathrm{ppt}$ from a starting concentration of 1,000 ppt. The treatment objective was 50 ppt. The sorption unit showed 10 ppt discharge after six months.
\end{abstract}

Laboratory-scale tests demonstrated the feasibility of sorbent regeneration. Results show that sorption behavior is not affected after four cycles. 


\section{TABLE OF CONTENTS}

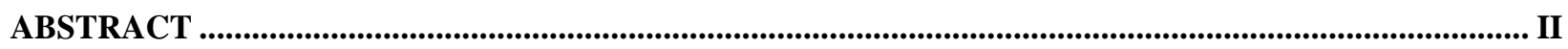

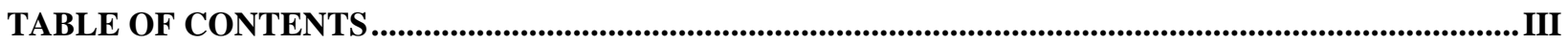

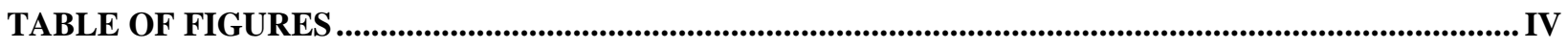

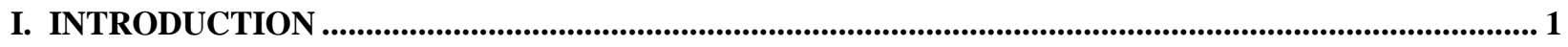

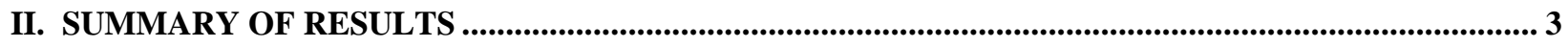

III. PREPARE AND TEST PILOT-SCALE QUANTITIES OF SORBENT ................................................ 6

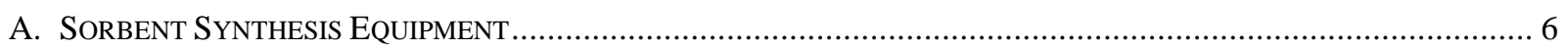

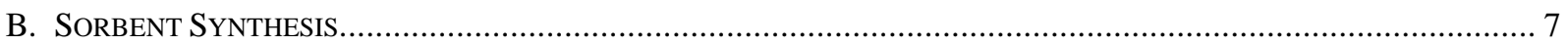

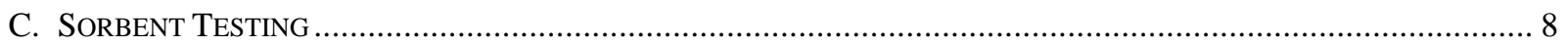

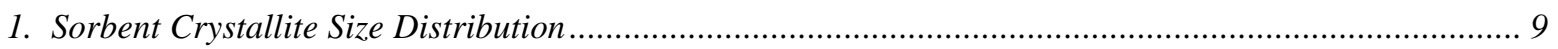

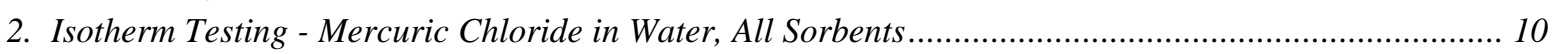

3. Isotherm Testing - Mercuric Chloride in Surrogate Wastewater, Three Sorbents.................................... 17

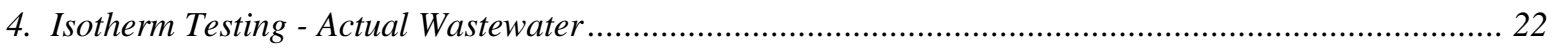

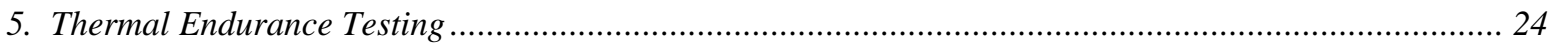

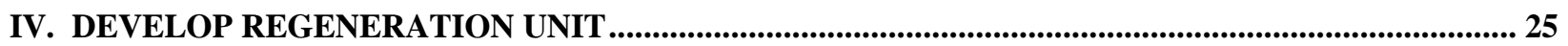

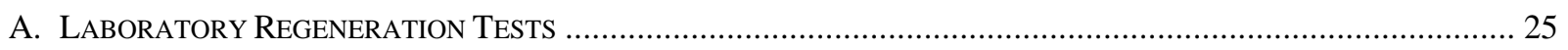

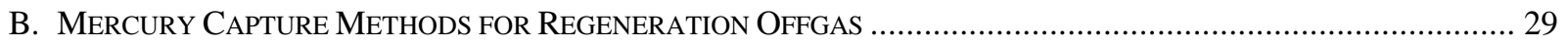

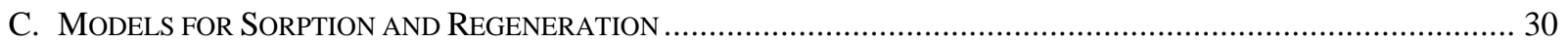

V. FIELD TEST OF SORPTION PROCESS........................................................................................................ 32

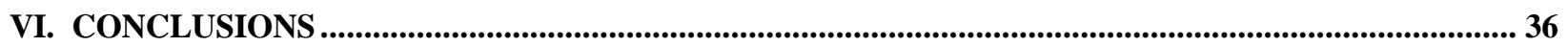

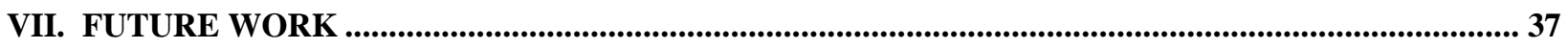

APPENDIX A. MODELS FOR SORPTION AND REGENERATION ......................................................... 38 


\section{TABLE OF FIGURES}

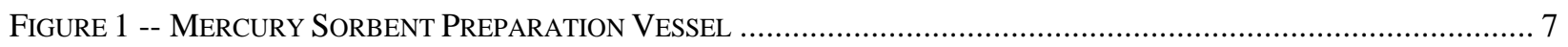

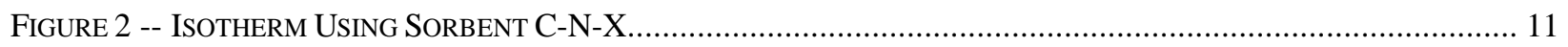

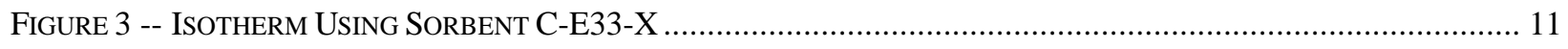

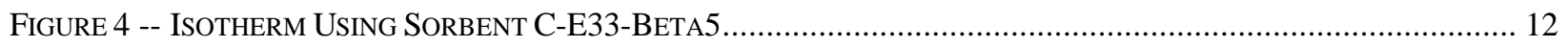

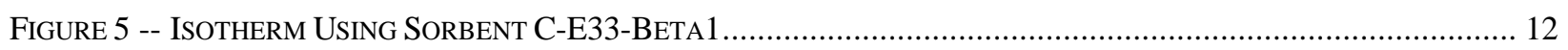

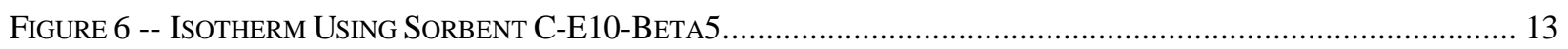

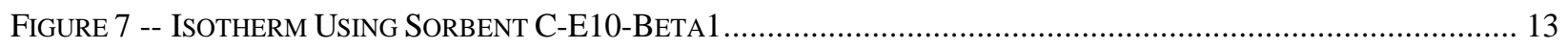

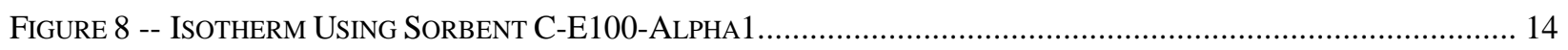

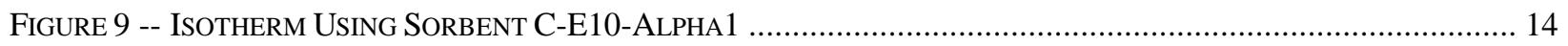

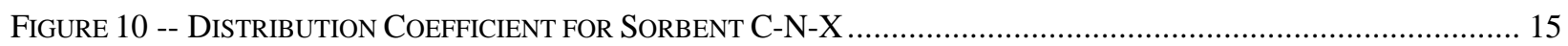

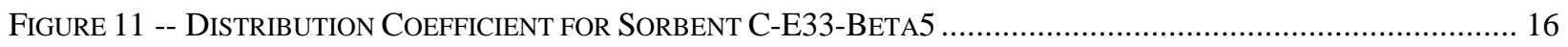

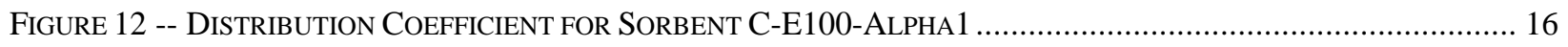

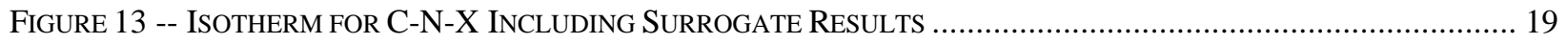

FiguRE 14 - Distribution COEFFICIENT FOR C-N-X INCLUding SURRogate RESUltS ..................................... 20

FigURE 15 -- ISOTHERM FOR C-E33-BETA5 InCLUding SURROGATE RESULTS ...................................................... 20

Figure 16 - Distribution CoefFicient For C-E33-Beta5 IncLuding SuRrogate Results.............................. 21

FiguRE 17 -- ISOTHERM FOR C-E100-ALPHA1 InCLUdING SURROGATE RESULTS .............................................. 21

FiguRE 18 -- Distribution COEFFICIENT FOR C-E100-AlPHA1 InCLUding SURROGATE RESUltS.......................... 22

FIGURE 19 -- ISOTHERM FOR SORBENT C-E100-ALPHAI INCLUDING OUTFALL 200 RESULTS................................... 23

FiguRE 20 -- Distribution COEFFICIENT FOR DATA IN FigURE 19 ...................................................................... 23

FIGURE 21 -- ISOTHERM RESULTS FROM REGENERATION TESTS ....................................................................... 28

Figure 22 -- Mercury Capture Tests Showing Impact of ScRUbber on VApor Phase Mercury

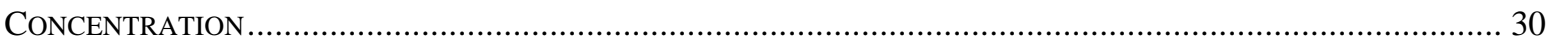

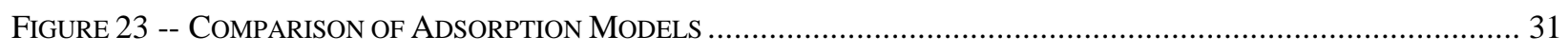

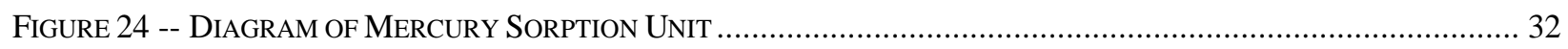

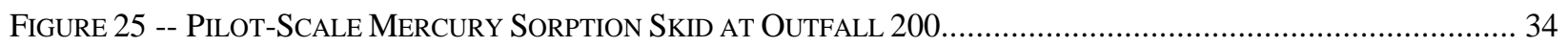

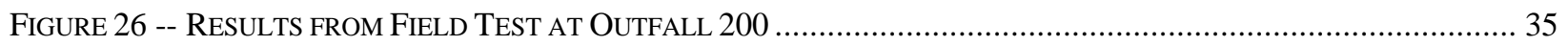

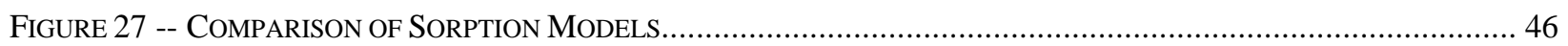




\section{INTRODUCTION}

The objective of this program is to develop and demonstrate a process for recovering mercury from contaminated liquid wastes at DOE sites. The technology addresses the need area identified as ESP-3, Toxic Component Removal. The technology will treat both the mercury-contaminated groundwater and primary liquid wastes already in DOE's inventory. In addition, the technology will treat mercury-contaminated secondary liquid wastes that will result from many of the processes and activities planned for treating the mercury-contaminated solids, soils, debris, and aqueous wastes in DOE's inventory.

ADA's process is based on the sorption of mercury on specialty sorbents and the regeneration of the sorbent by thermal means, recovering the desorbed mercury as liquid, elemental mercury. The sorbent technology uses noble metals on a porous substrate. ADA holds a patent on a similar process for removing mercury from off-gases (US 5,409,522, April 25, 1995) and has tested it under conditions typical of municipal waste incinerators. In this process, the sorbent is regenerated thermally, and the mercury is recovered for commercial recycle. ADA has adopted the name "Mercu-RE" to describe its process.

The technical objective of the proposed program is to demonstrate a turn-key, integrated version of ADA's mercury recovery process by treating a liquid waste at a DOE site. Achievement of this technical objective will make it possible for ADA to offer turn-key systems to a variety of commercial customers as well as to DOE contractors responsible for waste treatment.

ADA had two subcontractors under the Base Contract: the University of North Dakota, Energy and Environmental Research Center (EERC) and the Oak Ridge National Laboratory (ORNL). ADA was responsible for synthesizing and testing reproducible sorbents, modeling the sorption and regeneration process, and confirming that the process development work proceeded in a manner that effectively utilized its sorbents. This oversight responsibility included providing EERC with ADA's experience with the sorbents. EERC was responsible for the testing on surrogate wastes as well as designing, building, installing, and troubleshooting the small field sorption unit. ORNL tested the best sorbents on actual DOE wastes. The sorbent with the best mercury removal capability was then tested in a pilot-scale unit at a DOE site. ORNL supported the field test and was responsible for analytical sampling.

The Base Contract work was divided into four tasks. These tasks represented a progression from the preparation of pilot-scale quantities of sorbent, to the development of favorable methods and process conditions for regenerating the sorbent, to the field testing of the sorption process at a DOE site. Also, a variety of technical and management reports were required throughout the Base Contract. 
This report is one of the deliverables of the Base Contract and consists of the following major sections:

- Summary of results

- Prepare and test pilot-scale quantities of sorbent

- Develop regeneration unit

- Field test of sorption process

- Conclusions

- Future work 


\section{SUMMARY OF RESULTS}

Under the Base Contract, seven sorbents were synthesized in duplicate batches and tested with mercuric chloride solutions, surrogate waste containing mercury, and actual DOE wastewater from Outfall 200 at the Y-12 Plant in Oak Ridge, Tennessee. Manufacture of one of these sorbents was scaled-up to10 kg batches with uniform qualities. One 10-kg batch is currently removing mercury from Outfall 200 in a mini-pilot skid fabricated under this Base Contract. This field test removed mercury from the East Fork Poplar Creek to 1 ppt from a starting concentration of approximately $1,000 \mathrm{ppt}$. The treatment objective is to remove mercury to below 50 ppt.

The ADA sorbents show the following characteristics based upon testing:

- At high mercury solution concentrations, the sorbents take up more than ten percent of the noble metal weight in mercury.

- Thermogravimetric analysis on the untreated and treated sorbents showed thermal stability in air up to at least $700^{\circ} \mathrm{F}$, which is the regeneration temperature. Thermal endurance tests, however, indicate that the sorbents will degrade over time in air. Therefore, ADA will use nitrogen during sorbent regeneration.

- Results from X-ray Diffraction Line Broadening (XRD) on all sorbent samples showed a metal crystallite size below $60 \mathrm{~nm}$ and good reproducibility between batches, especially at low metal concentrations. Noble metal crystallite size for the sorbents was calculated between 20 and $38 \mathrm{~nm}$, which indicates an excellent degree of dispersion of the noble metal on the substrate.

- The results from the duplicate isotherm batches show reproducibility at the near- $1 \mathrm{~kg}$ level. Isotherm results from UND-EERC show similar mercury loading numbers for the sorbents when compared with the ADA isotherm tests.

- ADA representatives visited ORNL to discuss available waste streams for sorbent testing. Outfall 200 and the NRWTP were targeted as having a high priority for treatment due to current and future regulations on discharge limits for mercury. A decision was made to set up the sorption and regeneration unit at Outfall 200 at the Y-12 plant at Oak Ridge, Tennessee during September. The focus for the rest of the Base Contract was then on the low-level mercury-containing streams.

- Isotherm testing was performed using a surrogate Non-Radioactive Wastewater Treatment Plant (NRWTP) water on sorbent C-E100-Alpha1. CVAFS analysis indicated that the starting concentration of $210 \mathrm{ppt}$ mercury was reduced to $0.5 \mathrm{ppt}$ in several sorbent samples.

- Isotherm testing using an surrogate Outfall 200 wastewater with sorbents C-E100-Alpha1 and C-E33-Beta5 was performed. The sorbents behaved in a similar fashion, exhibiting excellent 
removal (>99.9\%) of mercuric chloride from the surrogate Outfall 200 liquid, resulting in a final mercury concentration of less than $1 \mathrm{ppt}$.

- Preparation of the triplicate $10-\mathrm{kg}$ batches of sorbent to prove reproducibility was successfully completed. Preparation of the 10-kg batch of sorbent C-E100-Alpha1, which is being used in the field tests, was also successfully completed, and laboratory tests showed that this large batch compared favorably with the smaller batches.

- Six regeneration tests were performed on ADA sorbent C-E100-Alpha1 at UND-EERC. The time required to completely remove mercuric chloride from the sorbent was longer than expected. Additional tests showed that the sorbent requires 52 hours to completely desorb mercuric chloride.

- Four sorption/regeneration cycles were performed on sorbent C-E100-Alpha1 at ADA's laboratory. For comparison, sorption/regeneration tests were also performed at UND-EERC. ADA determined that the time required to completely remove mercuric chloride from the sorbent was on the order of 52 hours, which matched the results from UND-EERC. The carbon-based sorbent performed very well after each regeneration, showing a removal of $99.9 \%$.

- Isotherms on actual waste containing mercury from Outfall 200 at Oak Ridge Tennessee were performed using an ADA sorbent (C-E100-Alpha1). These tests show mercury removal to concentrations below 1 ppt on actual DOE water.

- Fabrication of the mini-pilot skid that was used for treatment of Outfall 200 water was completed at UND-EERC. The pump, filter, and inlet lines were set up at Outfall 200 in August and liquid flowed through the lines to condition them. Tests showed that the lines (without sorbent) did not remove mercury from the stream. Field testing of the ADA sorbent at Outfall 200 began in September 1997. Results from the flow tests show a removal to $1 \mathrm{ppt}$ from a starting concentration of about 1,000 ppt. The treatment objective was $50 \mathrm{ppt}$.

Table 1 shows the milestones accomplished under this Base Contract. All milestones were completed on schedule. 
Table 1 -- Milestone Log for Contract DE-AC21-97MC32195

\begin{tabular}{|c|c|c|c|c|}
\hline $\begin{array}{l}\text { Element } \\
\text { Code }\end{array}$ & Description & $\begin{array}{l}\text { Planned } \\
\text { Completion } \\
\text { Date }\end{array}$ & $\begin{array}{c}\text { Actual } \\
\text { Completion } \\
\text { Date }\end{array}$ & Comments \\
\hline $1.1 \mathrm{a}$ & $\begin{array}{l}\text { Reproducible } 100 \text { gram } \\
\text { batches (ADA) }\end{array}$ & 6/30/97 & $5 / 97$ & $\begin{array}{l}\text { Reproducibility } \\
\text { confirmed from } \\
\text { isotherm data }\end{array}$ \\
\hline $1.1 \mathrm{~b}$ & $\begin{array}{c}\text { Three Sorbents Exceed } \\
\text { Performance Target on } \\
\text { Surrogate Wastes } \\
\text { (EERC) }\end{array}$ & $7 / 30 / 97$ & $5 / 97$ & $\begin{array}{c}\text { Tests performed at } \\
\text { Frontier Geosciences } \\
\text { on "Surrogate" } \\
\text { NRWTP water }\end{array}$ \\
\hline $1.1 \mathrm{c}$ & $\begin{array}{c}\text { Reproducible } 1 \mathrm{~kg} \\
\text { batches (ADA) }\end{array}$ & $6 / 30 / 97$ & $5 / 97$ & $\begin{array}{l}\text { Reproducibility } \\
\text { confirmed from } \\
\text { isotherm data }\end{array}$ \\
\hline $1.1 \mathrm{~d}$ & $\begin{array}{l}\text { Reproducible } 10 \mathrm{~kg} \\
\text { batches (ADA) }\end{array}$ & $8 / 31 / 97$ & $8 / 97$ & $\begin{array}{l}\text { Reproducibility } \\
\text { confirmed from } \\
\text { isotherm data }\end{array}$ \\
\hline $1.1 \mathrm{e}$ & $\begin{array}{l}\text { Two Sorbents Exceed } \\
\text { Performance Target on } \\
\text { Actual Waste (ORNL) }\end{array}$ & $7 / 31 / 97$ & 7/97 & $\begin{array}{l}\text { Only one sorbent will } \\
\text { be tested on actual } \\
\text { waste due to time } \\
\text { constraints }\end{array}$ \\
\hline $1.2 \mathrm{a}$ & $\begin{array}{l}\text { Develop Operational } \\
\text { Parameters for } \\
\text { Regeneration Unit } \\
\text { (EERC) }\end{array}$ & $9 / 30 / 97$ & $9 / 97$ & $\begin{array}{l}\text { Sorbent requires } 52 \\
\text { hours for complete } \\
\text { regeneration }\end{array}$ \\
\hline $1.3 \mathrm{a}$ & $\begin{array}{l}\text { Start Field Sorption } \\
\text { Testing }\end{array}$ & $10 / 31 / 97$ & 9/8/97 & $\begin{array}{l}\text { Discharge limit of less } \\
\text { than } 1 \mathrm{ppt} \text { achieved }\end{array}$ \\
\hline
\end{tabular}




\section{PREPARE AND TEST PILOT-SCALE QUANTITIES OF SORBENT}

The main objective of this task was to make kilogram quantities of sorbent with favorable, uniform properties. Specific objectives included the synthesis of duplicate 1-kg batches of sorbent that sorb more than ten percent of the noble metal weight in mercury, have noble metal crystallites less than $60 \mathrm{~nm}$ in diameter, and remove mercury from solution in a reproducible manner. The sorbents were first tested using mercuric chloride in water, and then the best three were tested using a surrogate waste. The best sorbent from the surrogate testing was then tested on actual DOE wastewater. The next objective in this task was to synthesize $10-\mathrm{kg}$ batches of sorbent with these same reproducible qualities.

This section describes the sorbent synthesis equipment, summarizes the synthesis method, and provides detailed results of sorbent testing.

\section{A. Sorbent Synthesis Equipment}

A vessel suitable for drying and calcining up to 1-kg batches of sorbent was fabricated at ADA (Figure 1). The vessel is four inches in diameter and eight inches in length. The vessel is constructed of 316 stainless steel and has ports for both inlet and outlet gas during drying and calcining of the sorbents. The whole vessel fits into an oven at ADA. During drying and calcining of the sorbent, gas was fed into the bottom of the vessel and then flowed upwards through the bed of sorbent. The temperature of the sorbent bed was monitored during heating.

The vessel used for preparation of the 10-kg batches of sorbent was also manufactured at ADA's fabrication shop. This vessel has a 14-inch inner diameter and a bed height of 14 inches, which results in a volume of 35.3 liters. When full of sorbent, the vessel was placed in a large oven and preheated gas was fed through a fitting at the bottom. The temperature of the sorbent bed was monitored during heating. 


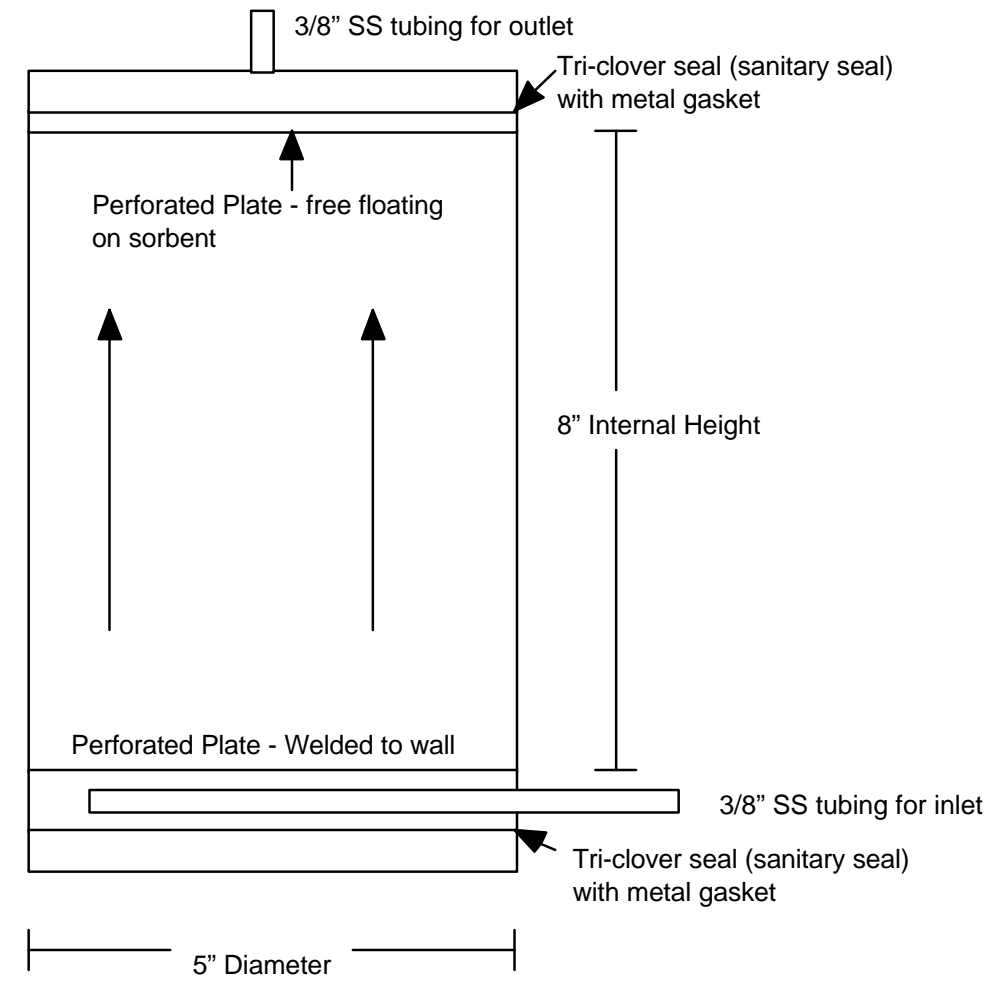

Figure 1 -- Mercury Sorbent Preparation Vessel

\section{B. Sorbent Synthesis}

The sorbents were prepared using a proprietary method developed by ADA. The basic method involved wetting a dry porous substrate, such as carbon or alumina, with a solvent containing the noble metal salt of interest. The wetted substrate was then dried and calcined at temperatures ranging from 300 to $400^{\circ} \mathrm{C}$.

The original plan was to prove reproducibility on 100-gram batches and then go to 1-kg followed by $10-\mathrm{kg}$ batches. All of the sorbents produced in the first set were at least 900 -gram batches to allow for UND-EERC testing. Because this was so close to the 1-kg value and there were no problems with reproducibility, ADA went from $0.9 \mathrm{~kg}$ to the $10-\mathrm{kg}$ batches.

Table 2 shows the sorbents prepared under the Base Contract.

Table 2 -- Sorbents Prepared and Tested by ADA 


\begin{tabular}{|c|c|c|}
\hline Sorbent Name & Batch Number & Batch Size \\
\hline C-N-X & & $1 \mathrm{~kg}$ \\
\hline C-E33-X & & $1 \mathrm{~kg}$ \\
\hline C-E33-Beta5 & $\# 1$ & $0.9 \mathrm{~kg}$ \\
\hline C-E33-Beta5-B2 & $\# 2$ & $0.9 \mathrm{~kg}$ \\
\hline C-E33-Beta1 & $\# 1$ & $0.9 \mathrm{~kg}$ \\
\hline C-E33-Beta1-B2 & $\# 2$ & $0.9 \mathrm{~kg}$ \\
\hline C-E10-Beta5 & $\# 1$ & $0.9 \mathrm{~kg}$ \\
\hline C-E10-Beta5-B2 & $\# 2$ & $0.9 \mathrm{~kg}$ \\
\hline C-E10-Beta1 & $\# 1$ & $0.9 \mathrm{~kg}$ \\
\hline C-E10-Beta1-B2 & $\# 2$ & $0.9 \mathrm{~kg}$ \\
\hline C-E100-Alpha1 & $\# 1$ & $0.9 \mathrm{~kg}$ \\
\hline C-E100-Alpha1-B2 & $\# 2$ & $0.9 \mathrm{~kg}$ \\
\hline C-E10-Alpha1 & $\# 1$ & $0.9 \mathrm{~kg}$ \\
\hline C-E10-Alpha1-B2 & $\# 2$ & $0.9 \mathrm{~kg}$ \\
\hline C-N-Beta1 & $\# 1$ & $10 \mathrm{~kg}$ \\
\hline C-N-Beta1 & $\# 2$ & $10 \mathrm{~kg}$ \\
\hline C-N-Beta1 & $\# 3$ & $10 \mathrm{~kg}$ \\
\hline C-E100-Alpha1 & & $10 \mathrm{~kg}$ \\
\hline
\end{tabular}

\section{Sorbent Testing}

This section describes the following laboratory-scale sorbent testing results: 
- Sorbent crystallite size distribution, all sorbents

- Isotherm tests - mercury chloride in water, all sorbents

- Isotherm tests - surrogate wastes, three sorbents

- Isotherm tests - actual wastes, one sorbent

- Thermal stability (three sorbents) and thermal endurance (one sorbent)

\section{Sorbent Crystallite Size Distribution}

X-Ray Diffraction Line Broadening (XRD) is an analytical method used to determine the size of metal crystallites. The peak placement as well as peak height and width are used to determine the type of metal and the crystal size.

XRD data was analyzed for all of the sorbents fabricated by ADA. Table 3 shows the results of these analyses. All of the sorbent samples had a crystallite size below $60 \mathrm{~nm}$, which was considered the maximum size for efficient use of the noble metal.

Sorbent C-E100-Alpha1 showed some variation between batches, but the small crystallite size probably contributes to error in the data reduction routine. Crystallite size is essentially the same between C-E100-Alpha1 and C-E10-Alpha1, indicating that these two preparation methods could be used interchangeably.

Sorbents C-E10-Beta1 and C-E33-Beta1 have the same crystallite sizes $(29-34 \mathrm{~nm})$, indicating that these two preparation methods are essentially the same. Sorbents C-E10-Beta5 and C-E33Beta5 show some variation between batches, but match each other in overall crystallite size. These two sorbents contain larger crystallites, although they are below the $60-\mathrm{nm}$ limit. These sorbents contain a higher percentage of noble metal than the other sorbents, indicating that there may be a limit to the amount of noble metal that can be deposited on the surface of the substrate without agglomeration of the crystallites. 
Table 3 -- X-Ray Diffraction Line Broadening Results

\begin{tabular}{|c|c|}
\hline Sorbent & Metal Crystallite Size (nm) \\
\hline C-E100-Alpha1 & 23.70 \\
\hline C-E100-Alpha1-B2 & 15.85 \\
\hline & \\
\hline C-E10-Alpha1 & 22.28 \\
\hline C-E10-Alpha1-B2 & 22.79 \\
\hline C-E10-Beta1 & 31.00 \\
\hline C-E10-Beta1-B2 & 29.48 \\
\hline & \\
\hline C-E10-Beta5 & 38.01 \\
\hline C-E10-Beta5-B2 & 45.93 \\
\hline & \\
\hline C-E33-Beta1 & 33.62 \\
\hline C-E33-Beta1-B2 & 31.61 \\
\hline & \\
\hline C-E33-Beta5 & 38.15 \\
\hline C-E33-Beta5-B2 & 45.38 \\
\hline
\end{tabular}

\section{Isotherm Testing - Mercuric Chloride in Water, All Sorbents}

Mercury sorption isotherms were measured on the sorbent samples using mercuric chloride. These isotherms were used to determine the ability of the sorbents to take up dissolved oxidized mercury from the liquid phase. ADA collected ten different mercury concentrations per isotherm ranging from $0.5 \mathrm{mg} / \mathrm{l}$ to $50 \mathrm{mg} / \mathrm{l}$ at equilibrium. This was considered the "standard" test for determining reproducibility of the mercury sorption capability of a particular sample.

The isotherms were performed with the sorbent and $100 \mathrm{ml}$ of mercuric chloride solution in capped flasks. The flasks were placed on a shaker table to ensure good contact between the solution and the sorbent. The flasks were agitated for 24 hours and filtered. The final and initial solutions were submitted for analysis. Duplicate isotherms were performed on one sorbent batch (C-E33-Beta1) to check the validity of the analysis.

Isotherm results were obtained from UND-EERC for three sorbents (C-N-X, C-E100-Alpha1, and $\mathrm{C}-\mathrm{E} 33-\mathrm{X})$ and compared with ADA data. These tests were performed using mercuric chloride in water as a check on the ADA analytical methods.

Isotherms are presented in Figures 2 - 9. The isotherms show good reproducibility of sorbent preparation and analytical methods. Only one isotherm (C-E100-Alpha1) showed significant data scatter, which may have been caused by analytical error. 


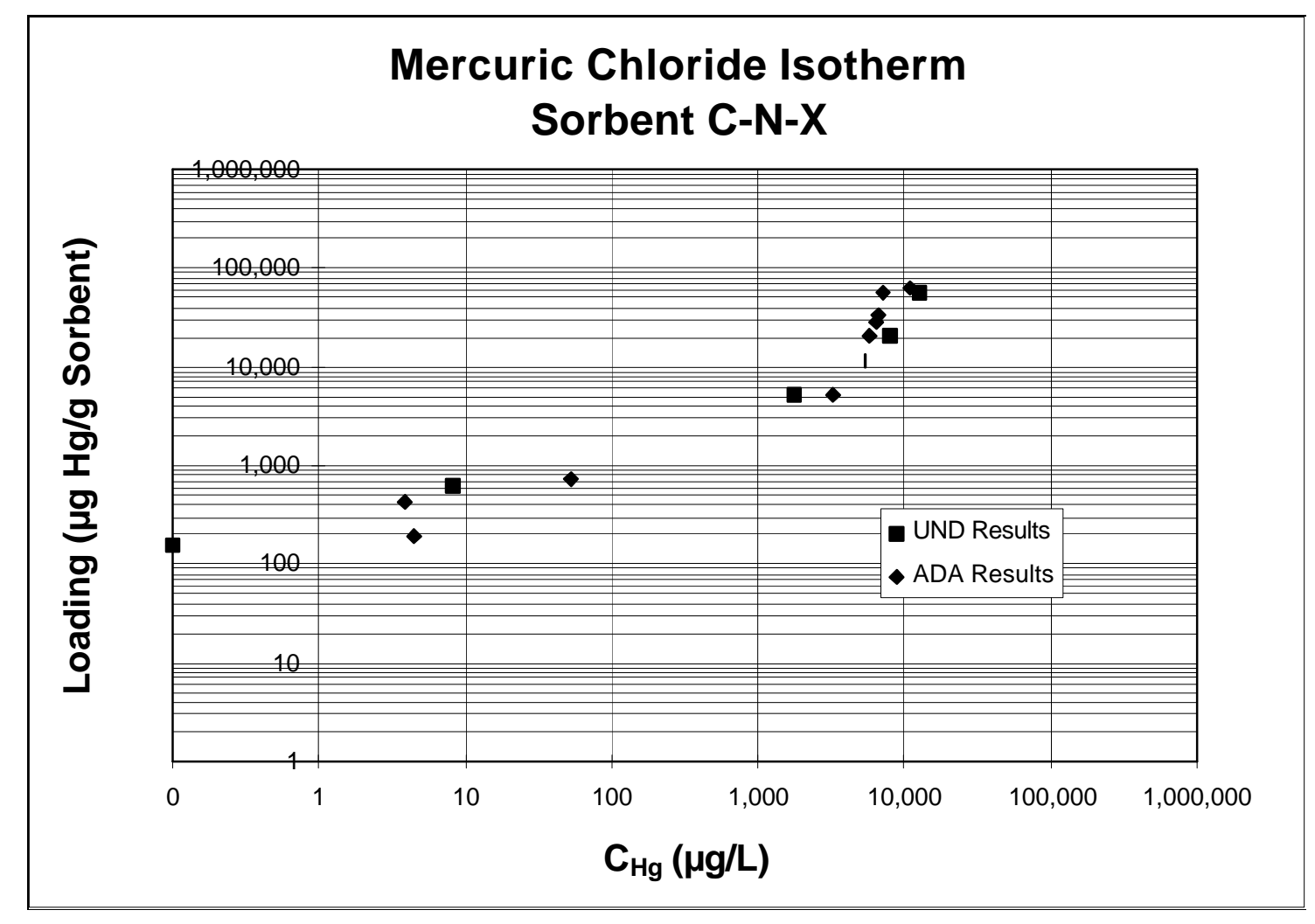

Figure 2 -- Isotherm Using Sorbent C-N-X

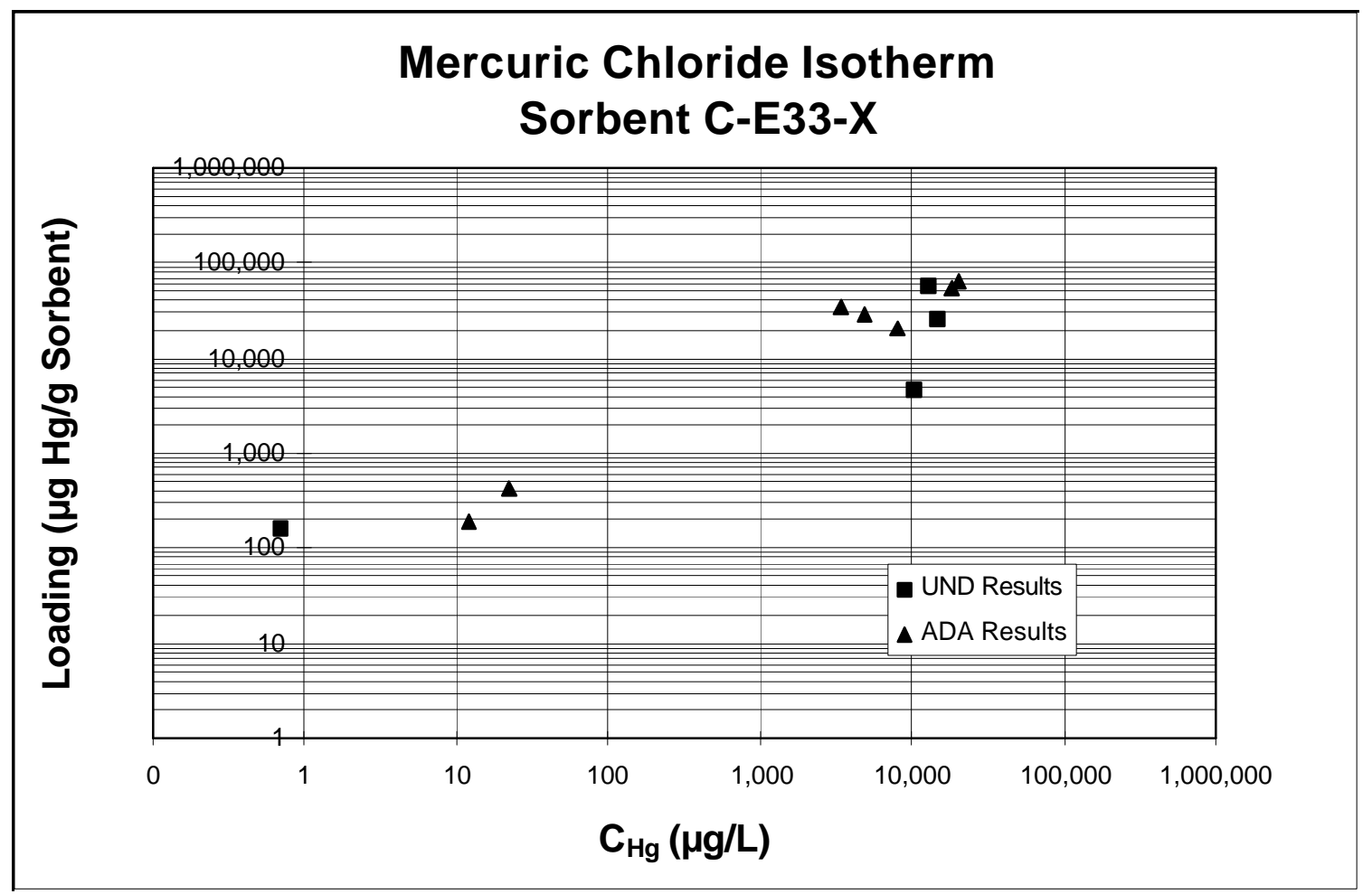

Figure 3 -- Isotherm Using Sorbent C-E33-X 


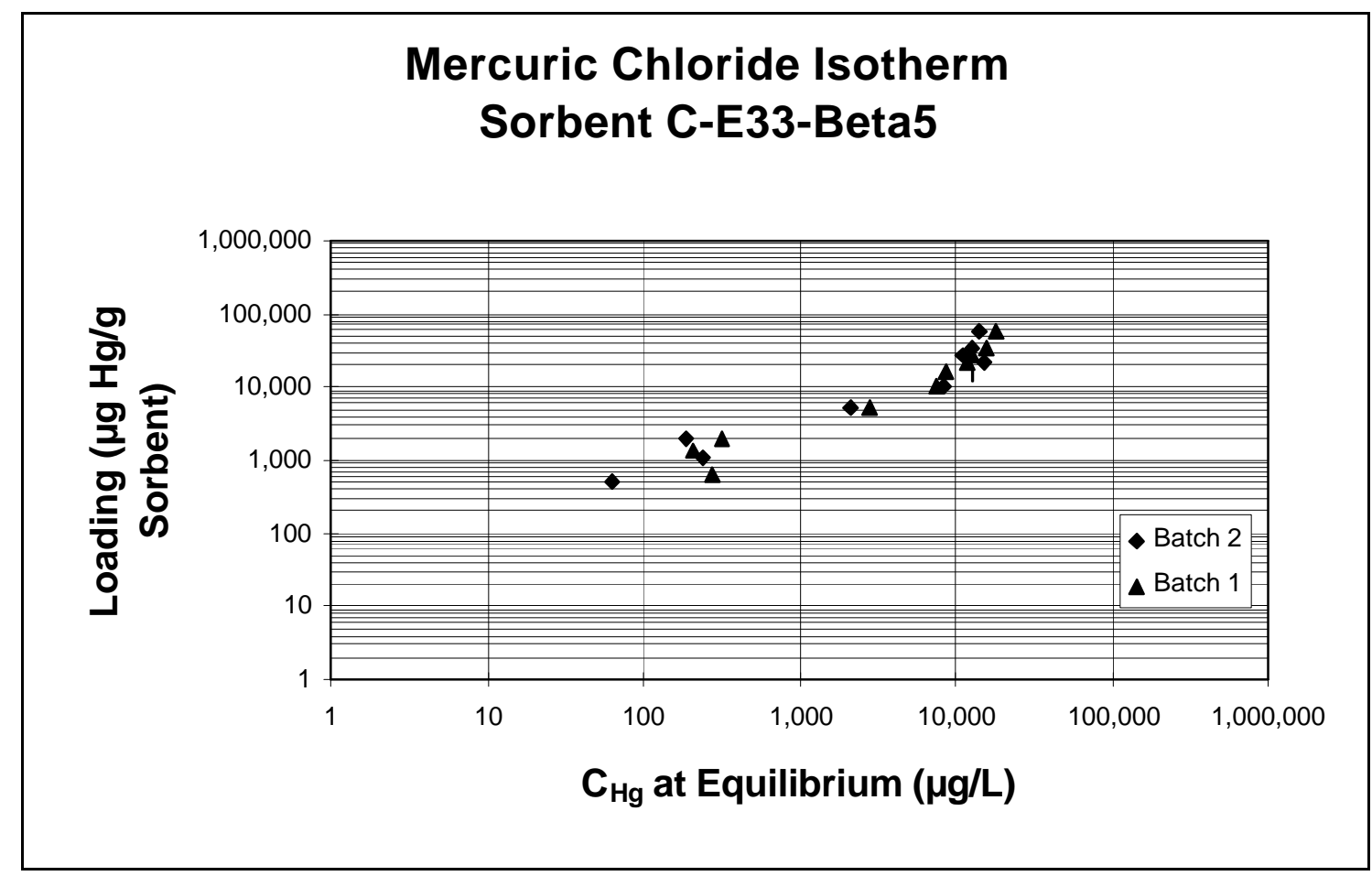

Figure 4 -- Isotherm Using Sorbent C-E33-Beta5

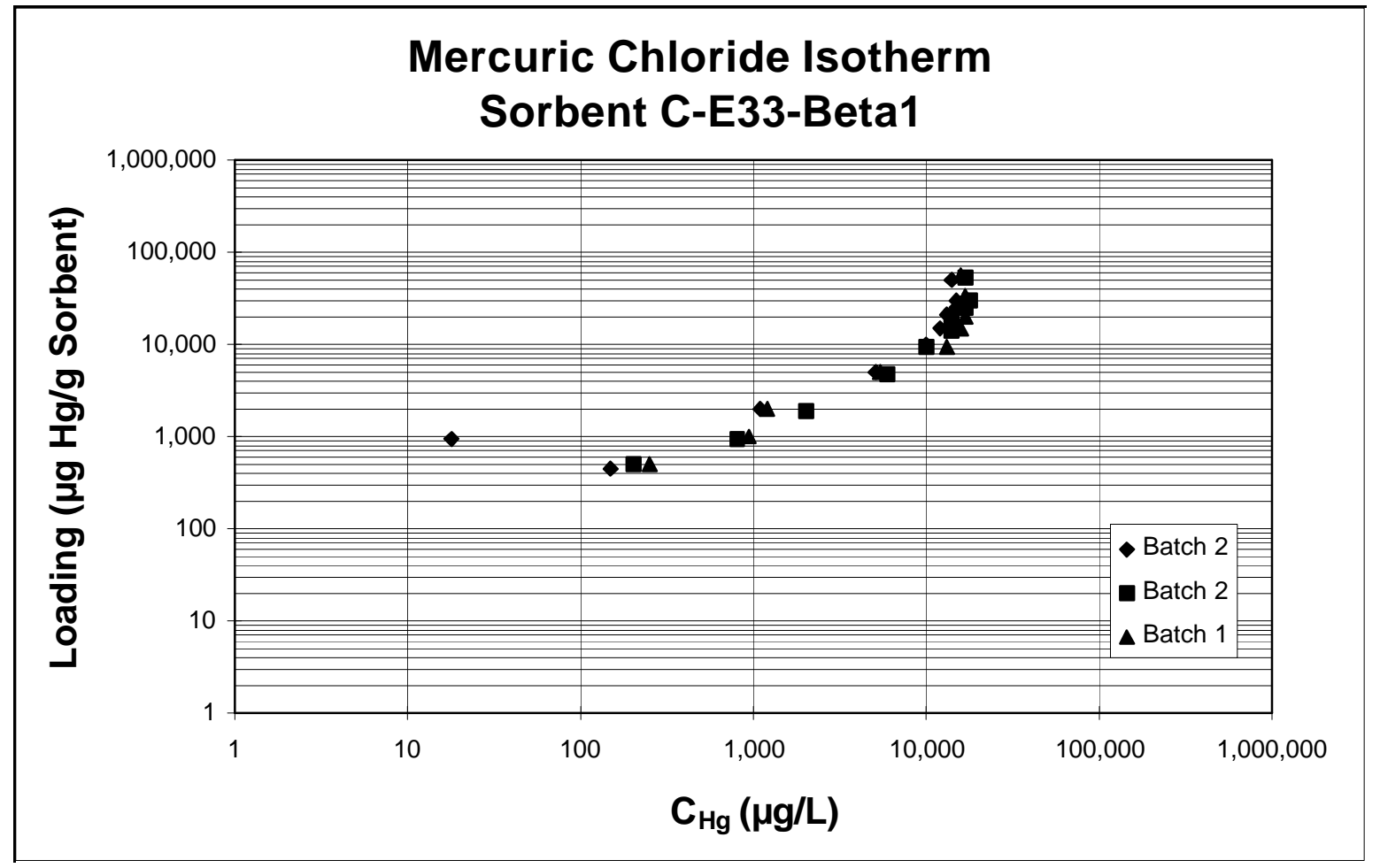

Figure 5 -- Isotherm Using Sorbent C-E33-Beta1 


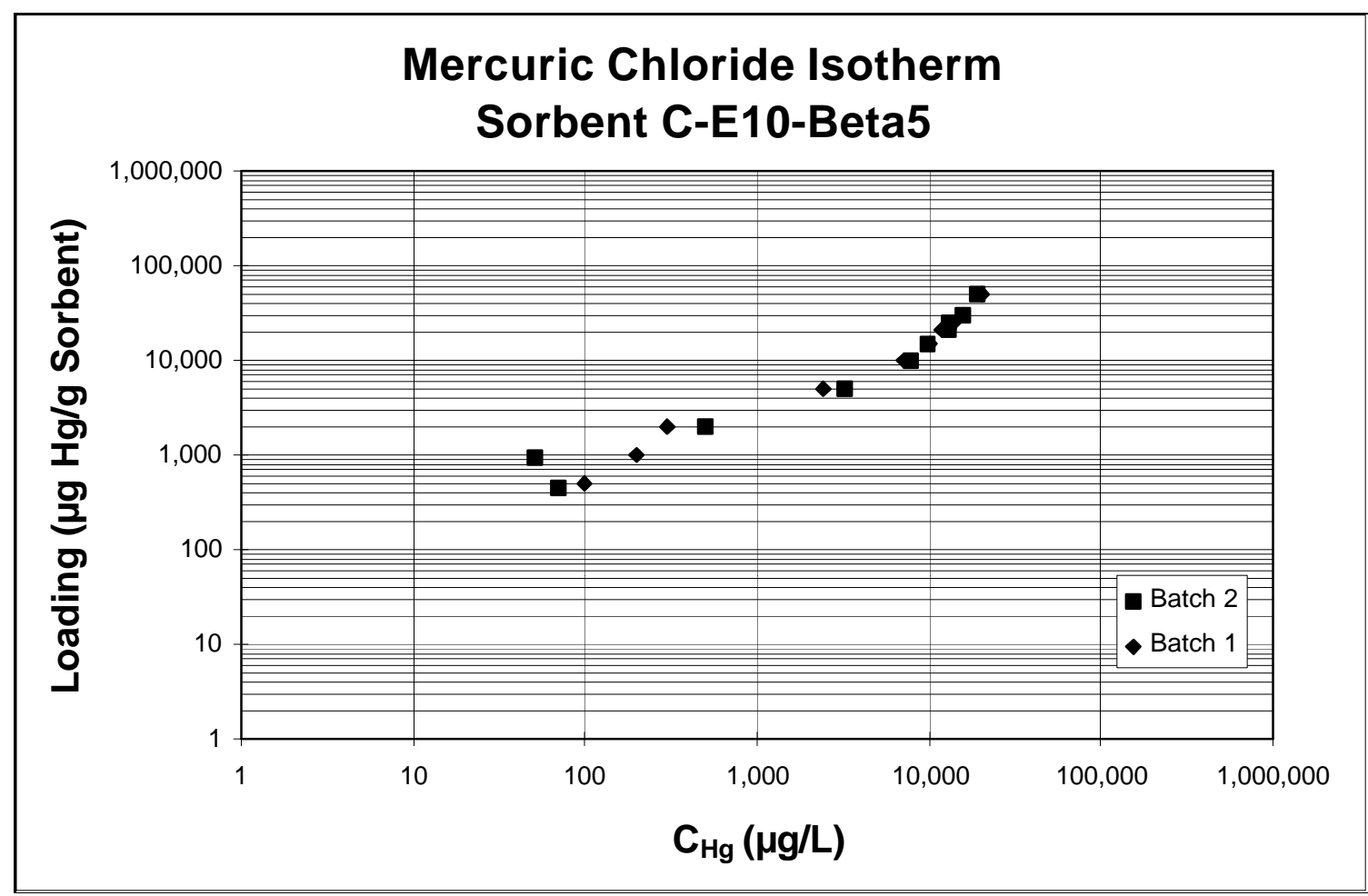

Figure 6 -- Isotherm Using Sorbent C-E10-Beta5

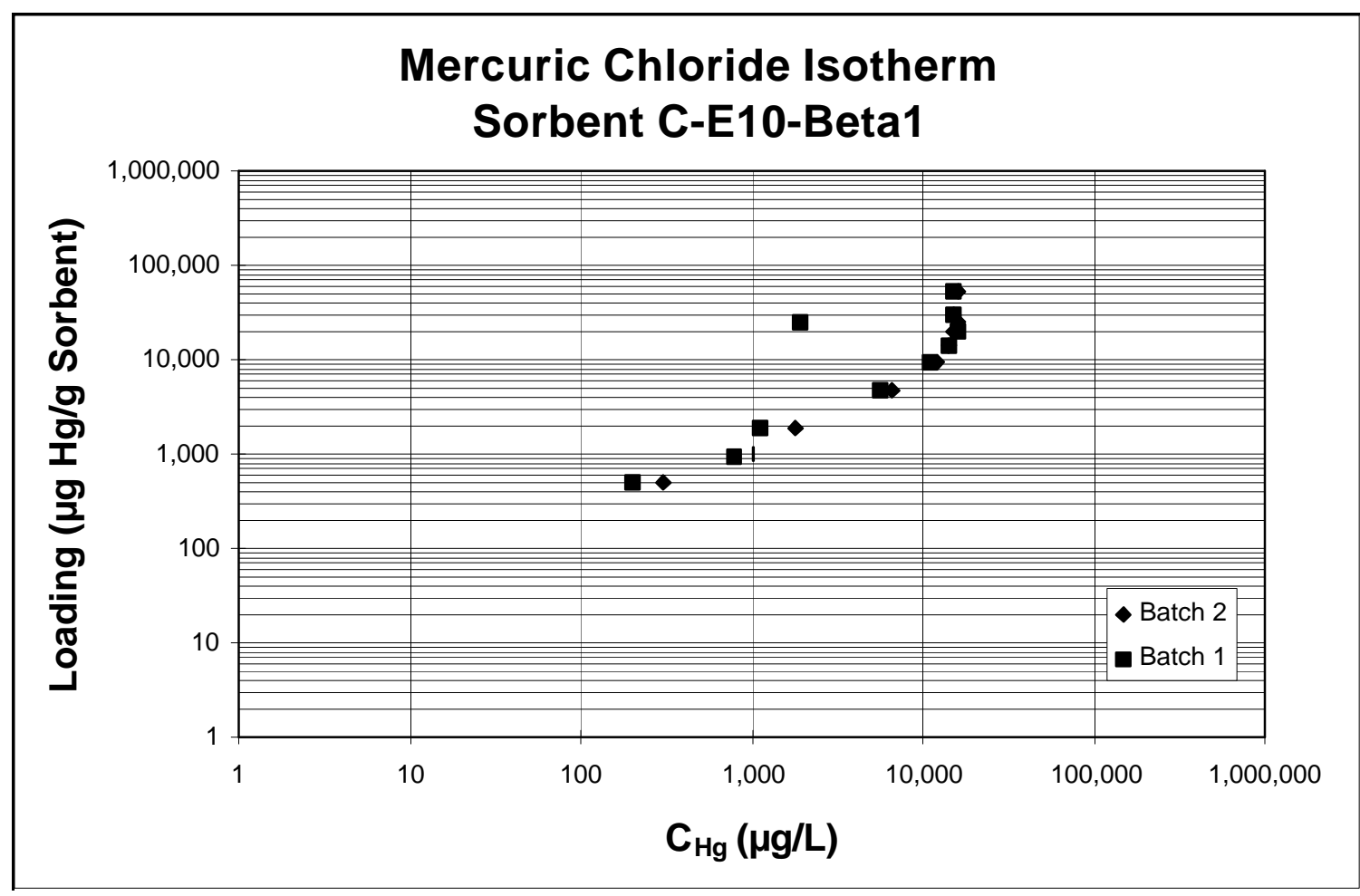

Figure 7 -- Isotherm Using Sorbent C-E10-Beta1 


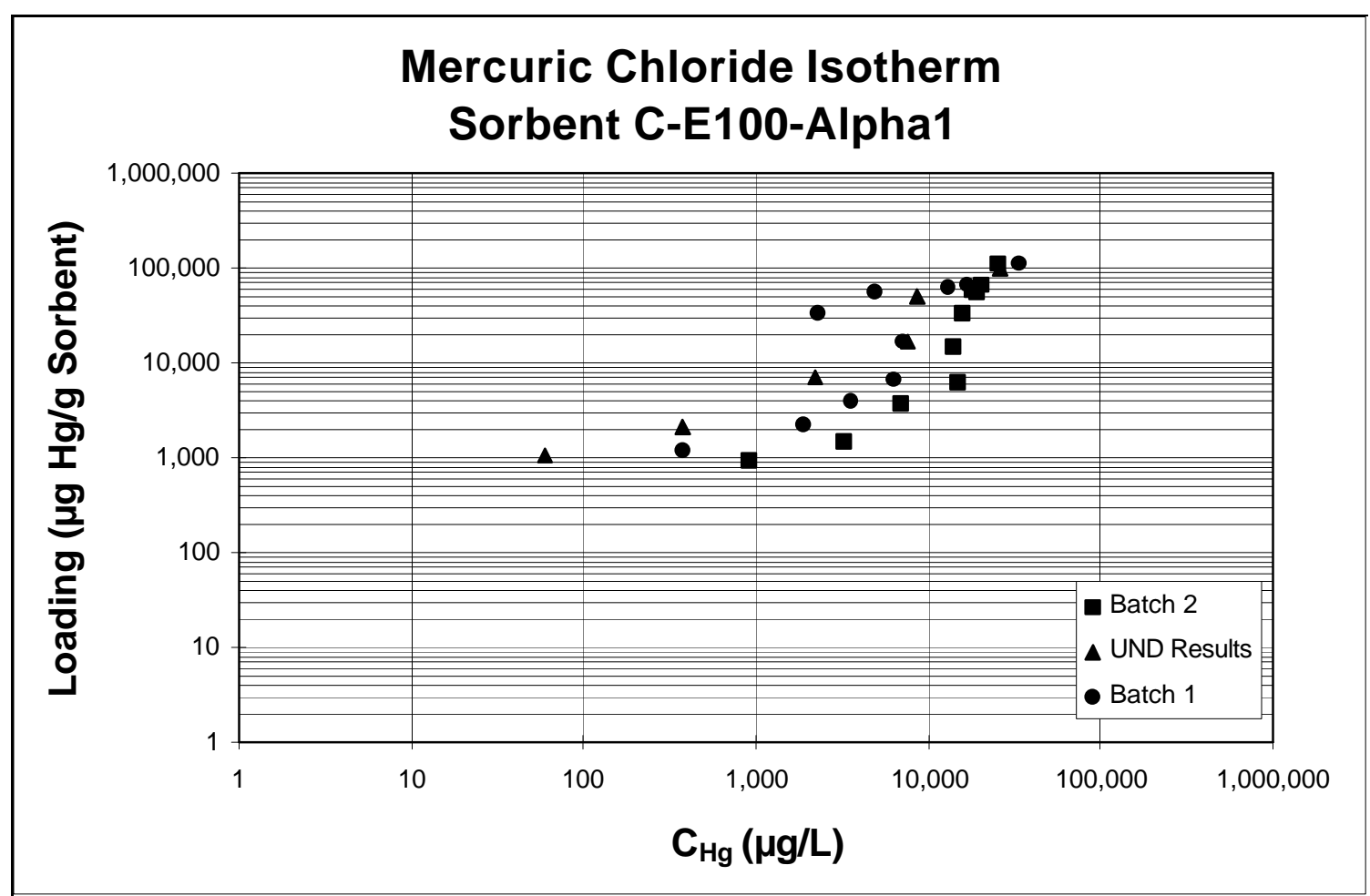

Figure 8 -- Isotherm Using Sorbent C-E100-Alpha1

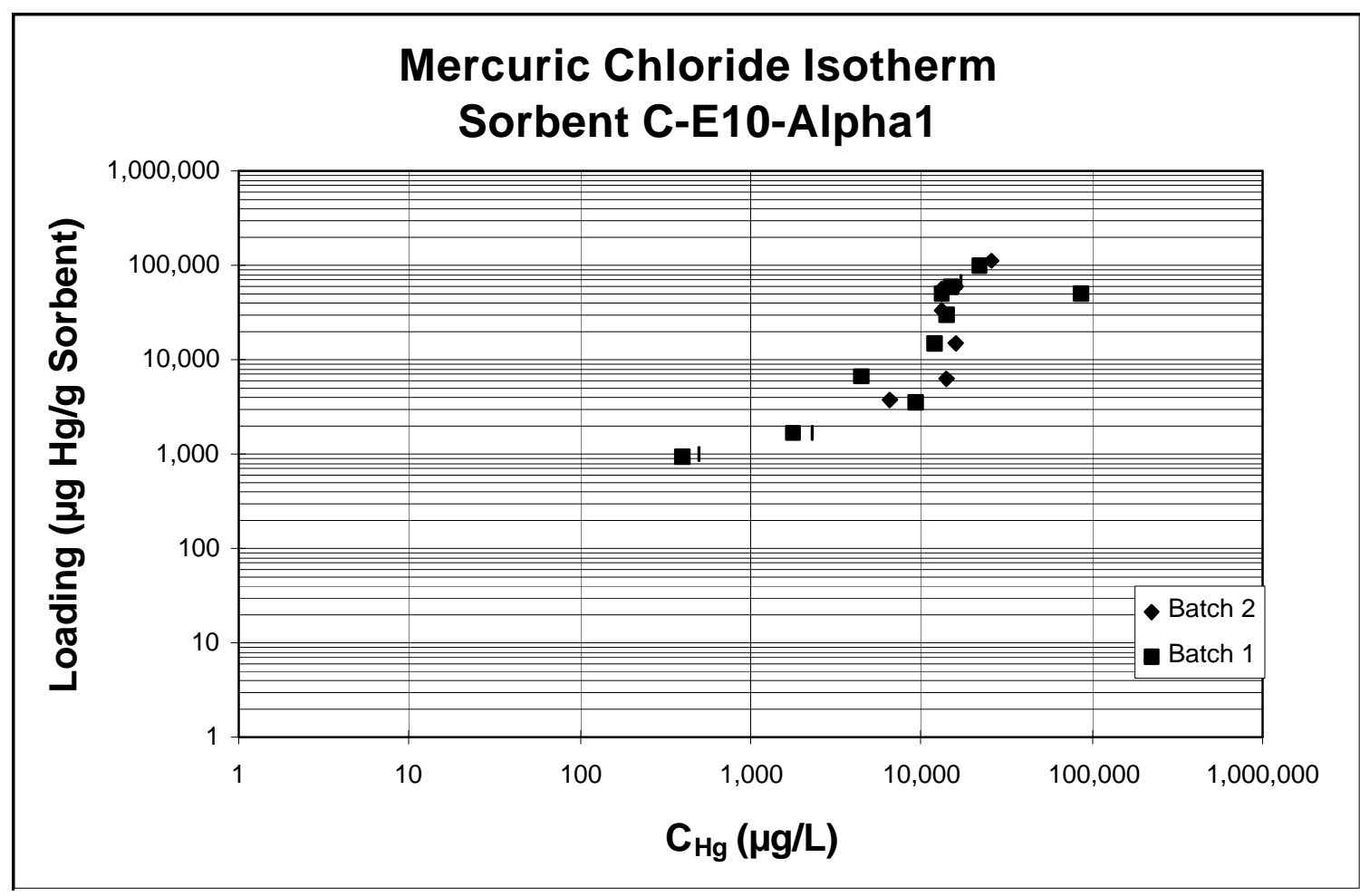

Figure 9 -- Isotherm Using Sorbent C-E10-Alpha1 
Distribution coefficients were determined for sorbents C-N-X, C-E100-Alpha1, and C-E33Beta5. The distribution coefficient is the amount of liquid at a specific mercury concentration that can be treated per mass of sorbent. Figures 10-12 show the distribution coefficients for the three sorbents. Sorbents C-N-X show a linear trend from less than $1 \mathrm{ppm}$ to about 5,000 ppm. The data for sorbent C-E100-Alpha1 show more scatter.

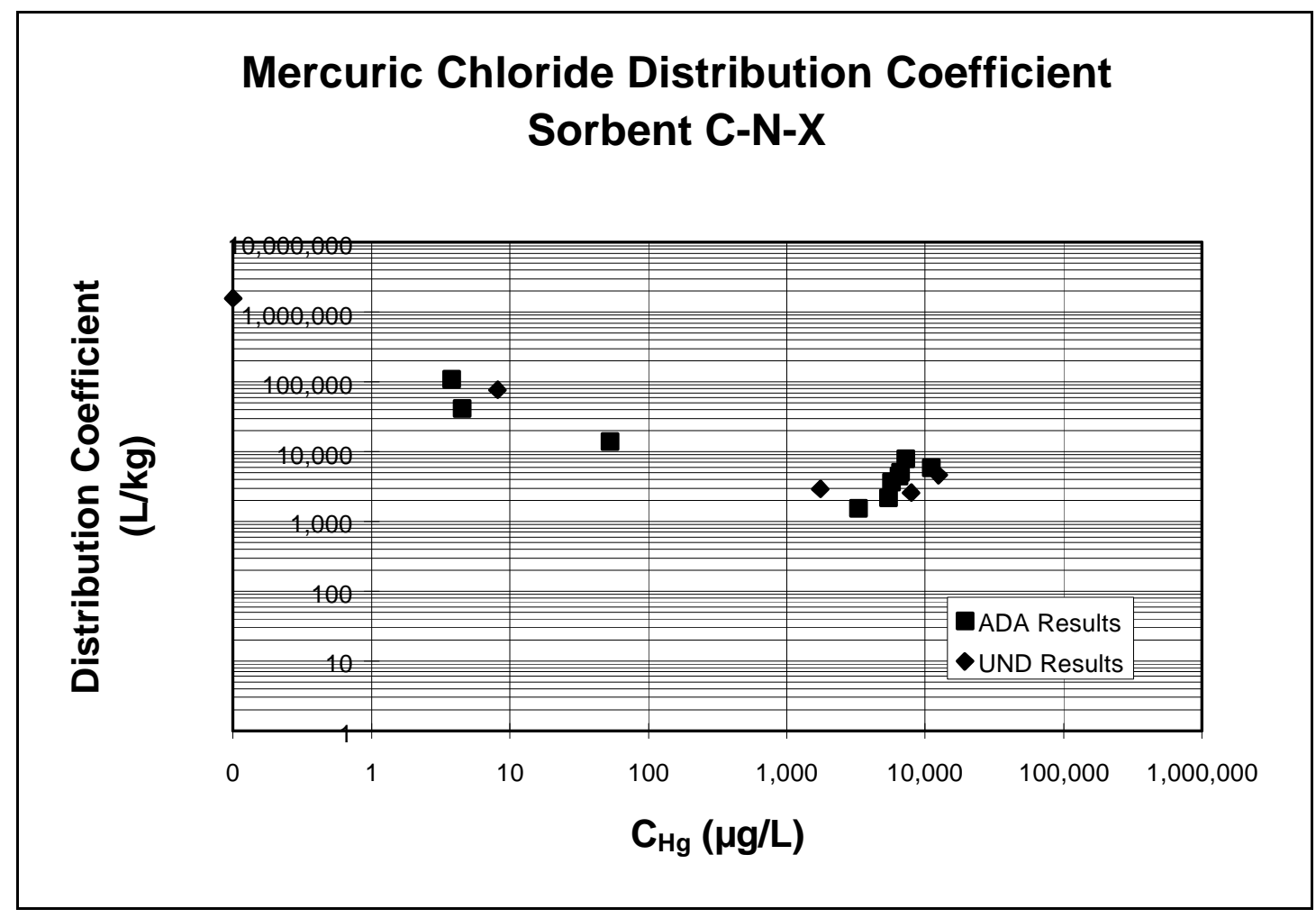

Figure 10 -- Distribution Coefficient for Sorbent C-N-X 


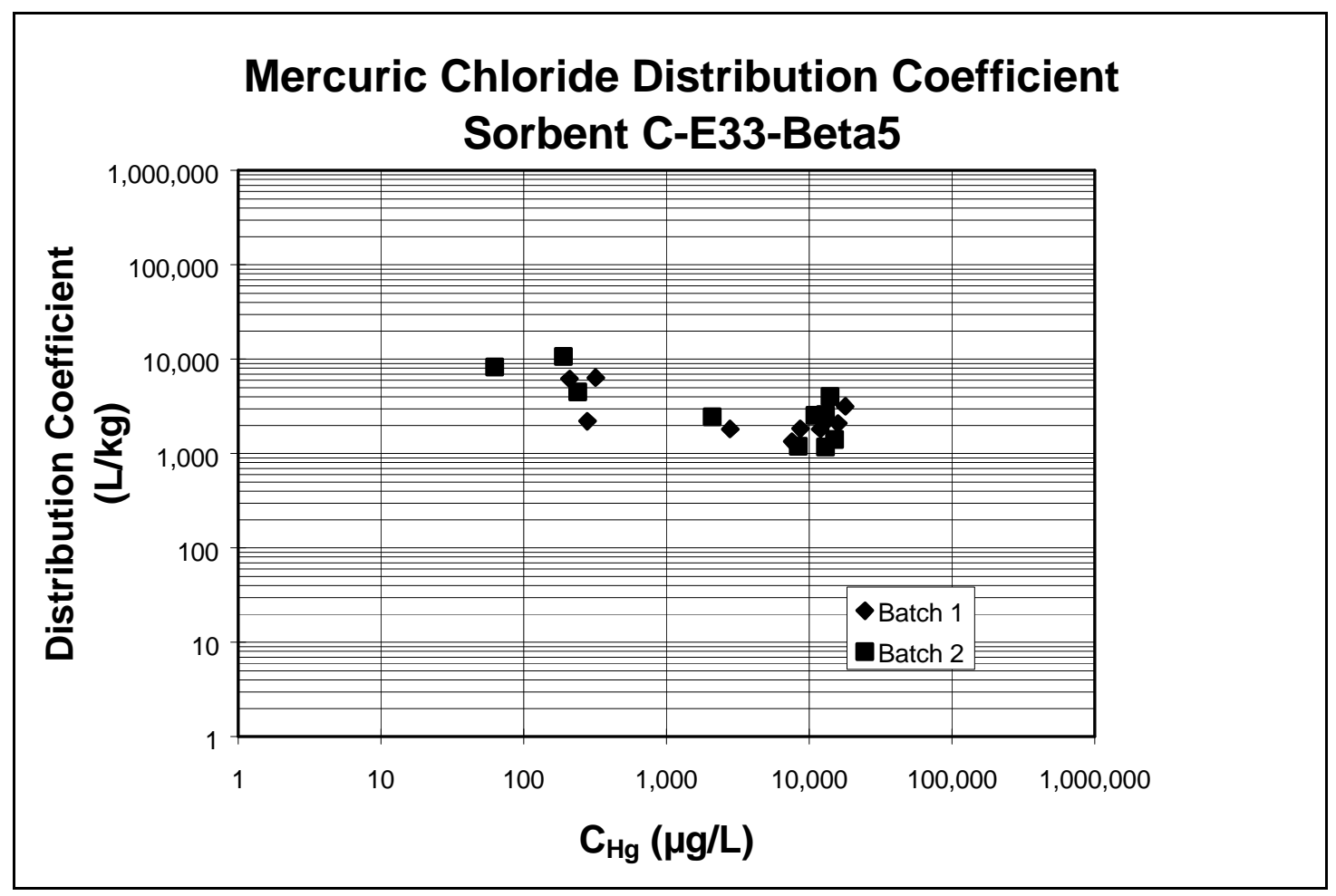

Figure 11 -- Distribution Coefficient for Sorbent C-E33-Beta5

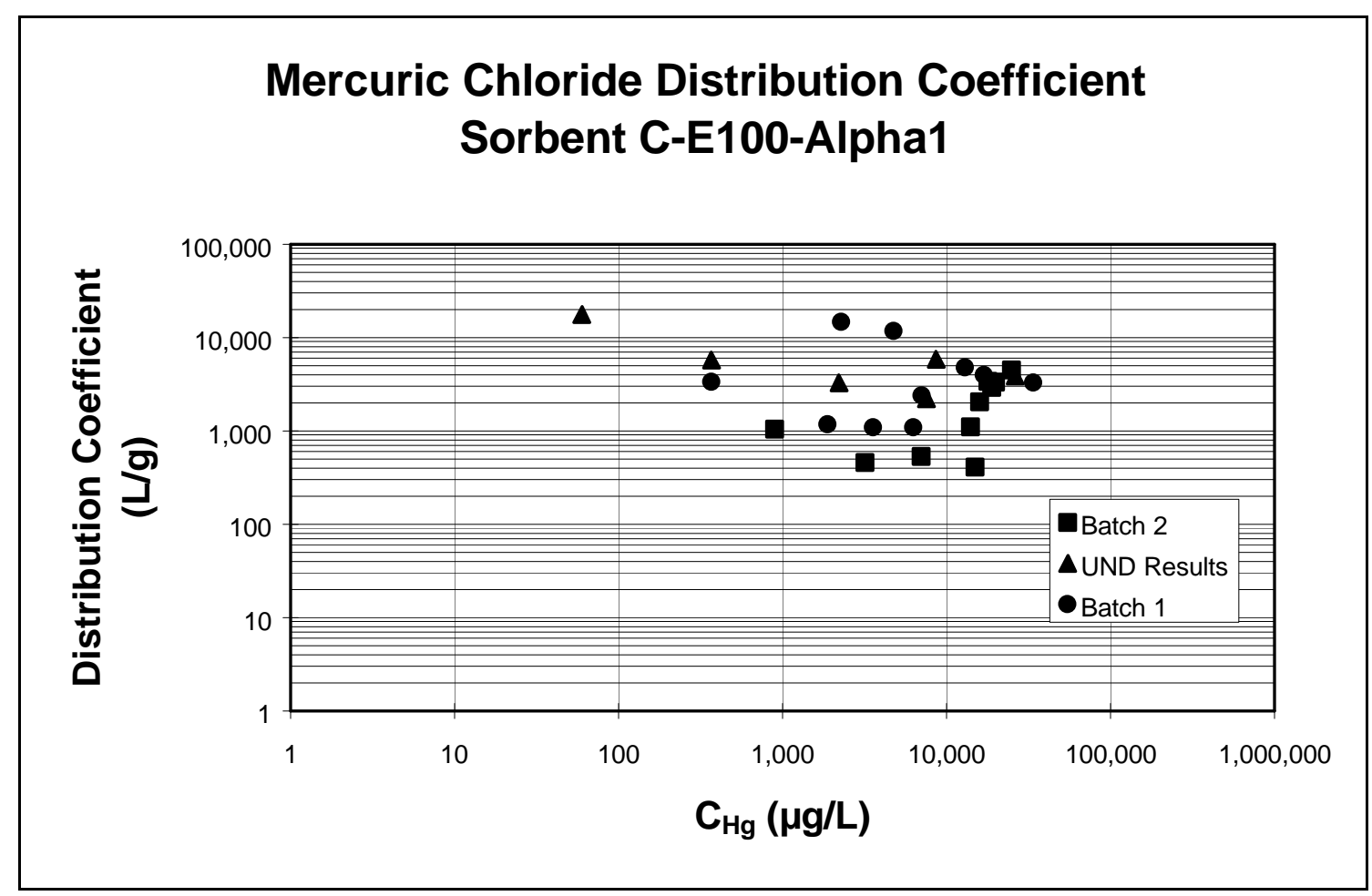

Figure 12 -- Distribution Coefficient for Sorbent C-E100-Alpha1 


\section{Isotherm Testing - Mercuric Chloride in Surrogate Wastewater, Three Sorbents}

Most of the isotherm tests were performed at high mercury concentrations in anticipation of treating high mercury wastes. Because of the low mercury concentration in Outfall 200 and NRWTP wastewaters at ORNL, additional tests were required to measure sorbent performance at ultra-low mercury concentrations. Three sorbents were sent to Frontier Geosciences for ultra-low level isotherm testing and analysis. The sorbents were C-N-X, C-E100-Alpha1 and C-E33-Beta5. Frontier performed the isotherms using a surrogate of the NRWTP waters, and then analyzed the samples using Cold Vapor Atomic Fluorescence (CVAFS).

The starting concentration of mercury was $210 \mathrm{ppt}$. The final concentration was $0.49 \mathrm{ppt}$ in one sample for sorbent C-N-X. The final concentration for sorbent C-E33-Beta5 was not as low as for sorbent $\mathrm{C}-\mathrm{N}-\mathrm{X}$. One data point was high $(81 \mathrm{ppt})$, which may have been caused by contamination of the sample. The final concentrations for sorbent C-E100-Alpha1 were 0.47 and 0.49 ppt. A duplicate was performed on one sample $(0.47 \mathrm{ppt})$, resulting in a value of 0.54 ppt. This shows that the results are reproducible. These data also show that the ADA sorbents can remove even low concentrations of mercury in water to levels below the most stringent regulatory requirements.

Isotherms were then performed on sorbents C-E100-Alpha1, C-E33-Beta5, and C-N-X using surrogate Outfall 200 water made at UND-EERC. The starting mercury concentration was 800 $\mathrm{ng} / \mathrm{L}$. The surrogate also had the same $\mathrm{pH}$ and contained the same quantities of the ionic species as the Y-12 Outfall 200 waste stream, with the exception of radioactive materials. In these tests, the volume and concentration of the liquid remained constant, while the amount of sorbent added to the liquid varied. A test matrix was developed to evaluate the sorption capability of each of the three sorbents over a sorbent weight range of five orders of magnitude. The test matrix included blank, duplicate and check samples for quality assurance/quality control purposes. Each sorbent was carefully weighed and combined with surrogate waste solution. The samples were agitated for $24 \mathrm{hr}$, after which they were filtered and the liquid shipped in a refrigerated cooler to Frontier Geosciences for analysis. At the laboratory, the samples were oxidized with $1 \%$ (v/v) $0.2 \mathrm{~N} \mathrm{BrCl}$ and analyzed by tin chloride reduction, dual gold amalgamation, and cold-vapor atomic fluorescence detection.

Table 4 shows the composition of the surrogate Outfall 200 water used in the tests performed at UND-EERC.

Table 4 -- Composition of Surrogate Outfall 200 Wastewater

\begin{tabular}{|c|c|}
\hline Ion & Concentration $(\mathbf{m g} / \mathbf{L})$ \\
\hline $\mathrm{Hg}^{+2}$ & 0.0008 \\
\hline $\mathrm{Mg}^{+2}$ & 10.21 \\
\hline
\end{tabular}




\begin{tabular}{|c|c|}
\hline $\mathrm{SO}_{4}^{-2}$ & 53 \\
\hline $\mathrm{K}^{+}$ & 1.7 \\
\hline $\mathrm{NO}_{3}^{-}$ & 6.9 \\
\hline $\mathrm{Ca}^{+2}$ & 49.92 \\
\hline $\mathrm{Cl}^{-}$ & 90.66 \\
\hline $\mathrm{Na}^{+}$ & 75.62 \\
\hline $\mathrm{HCO}_{3}^{-}$ & 200.687 \\
\hline
\end{tabular}

A review of the $\mathrm{QA} / \mathrm{QC}$ samples indicated that the analytical results obtained reasonably represent the sorbent behavior during the adsorption isotherm tests. While reproducibility tended to be erratic, possibly because the $\mathrm{BrCl}$ preservative was not added to the samples prior to their shipment, it does not appear that the experimental results were significantly compromised by the sample preparation.

The mercury adsorption capabilities of the three sorbents tested (C-E100-Alpha1, C-E33-Beta5 and $\mathrm{C}-\mathrm{N}-\mathrm{X}$ ) were very similar. All three were shown to be capable of removing $>99.9 \%$ of the mercury present in the surrogate wastewater. All three sorbents removed mercury to below 1 ppt at the highest amount of sorbent $(5 \mathrm{~g})$. All three sorbents removed mercury to below 12 ppt at $0.5 \mathrm{~g}$ sorbent. Only C-E100-Alpha1 removed mercury below $12 \mathrm{ppt}$ using $0.05 \mathrm{~g}$ sorbent.

The isotherm data were fitted to several isotherm models in order to find which model best described the sorbent behavior. Each set of data contained a data point that was outside of the apparent pattern shown by the other samples. This made it difficult to determine the shape of the sorption curve. Most of the data fitted Henry's Law, Langmuir, and Redlich-Peterson equations for sorbent behavior. When the data were fitted to a Freundlich model, sorbent C-E33-Beta5 showed the least desirable sorption behavior, although all three sorbents behaved very well at these low concentrations.

Figures 13-18 show the isotherms and corresponding distribution coefficients including the UNDEERC data with the surrogate test results. Figure 13 shows sorbent $\mathrm{C}-\mathrm{N}-\mathrm{X}$ based upon the loading of mercury on the sorbent. The data fit in very well with previous test results, and fill in the curve between the other data points. Figure 14 shows the same data with the calculation for the distribution coefficient. This shows a relatively linear behavior in the range tested.

Figure 15 shows sorbent C-E33-Beta5 based upon the loading of mercury on the sorbent. The data does not have as good of a fit with previous test results. Figure 16 shows the same data with 
the calculation for the distribution coefficient. These data are also outside the pattern of the previous results.

Figure 17 shows the results with sorbent C-E100-Alpha1 based upon the loading of mercury on the sorbent. These data also fit in very well with previous test results and fill in the curve between the other data points. Figure 18 shows the same data with the calculation for the distribution coefficient. Because of the amount of scatter in the data, it is difficult to determine the slope of the curve.

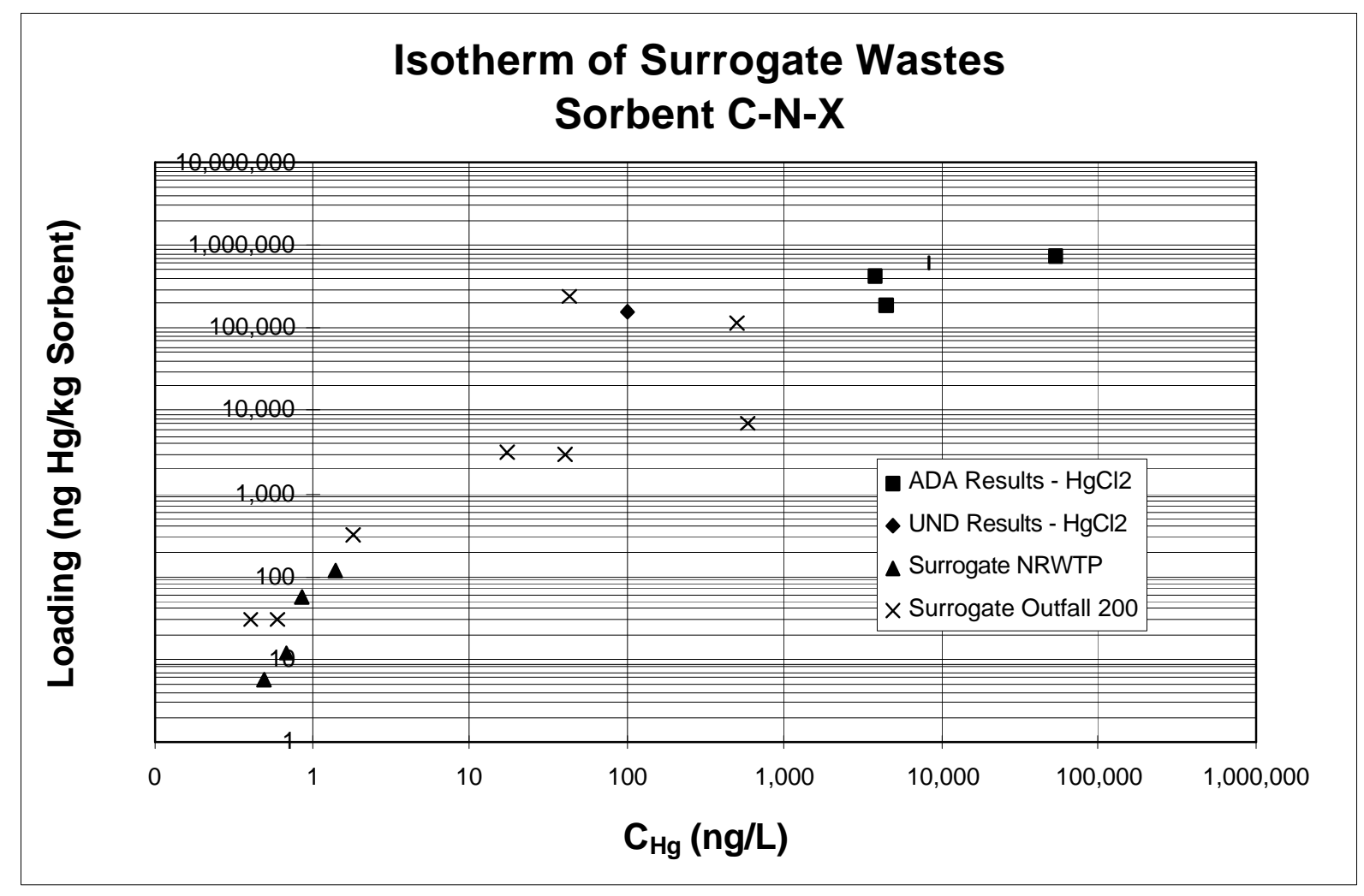

Figure 13 -- Isotherm for C-N-X Including Surrogate Results 


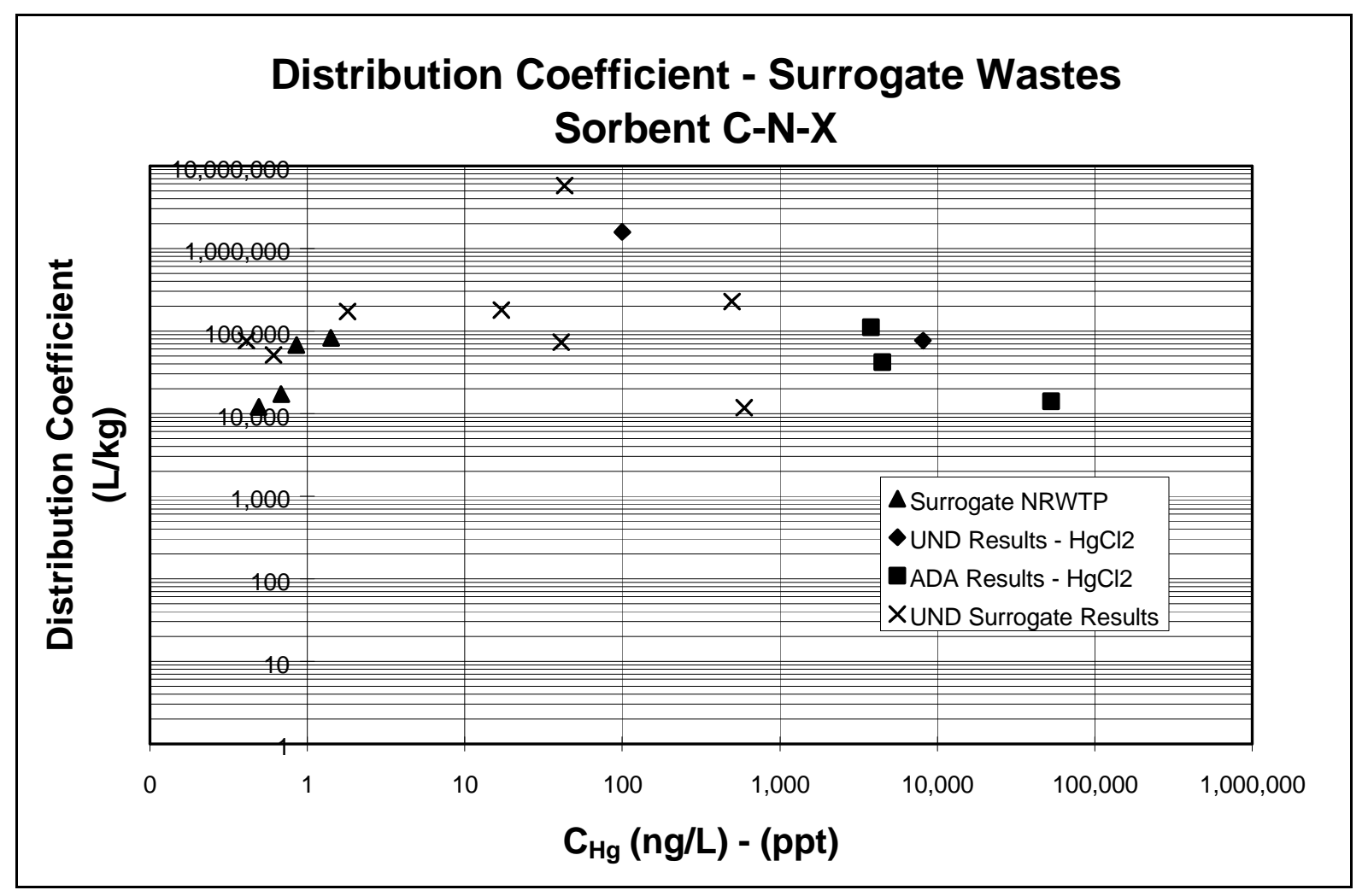

Figure 14 - Distribution Coefficient for C-N-X Including Surrogate Results

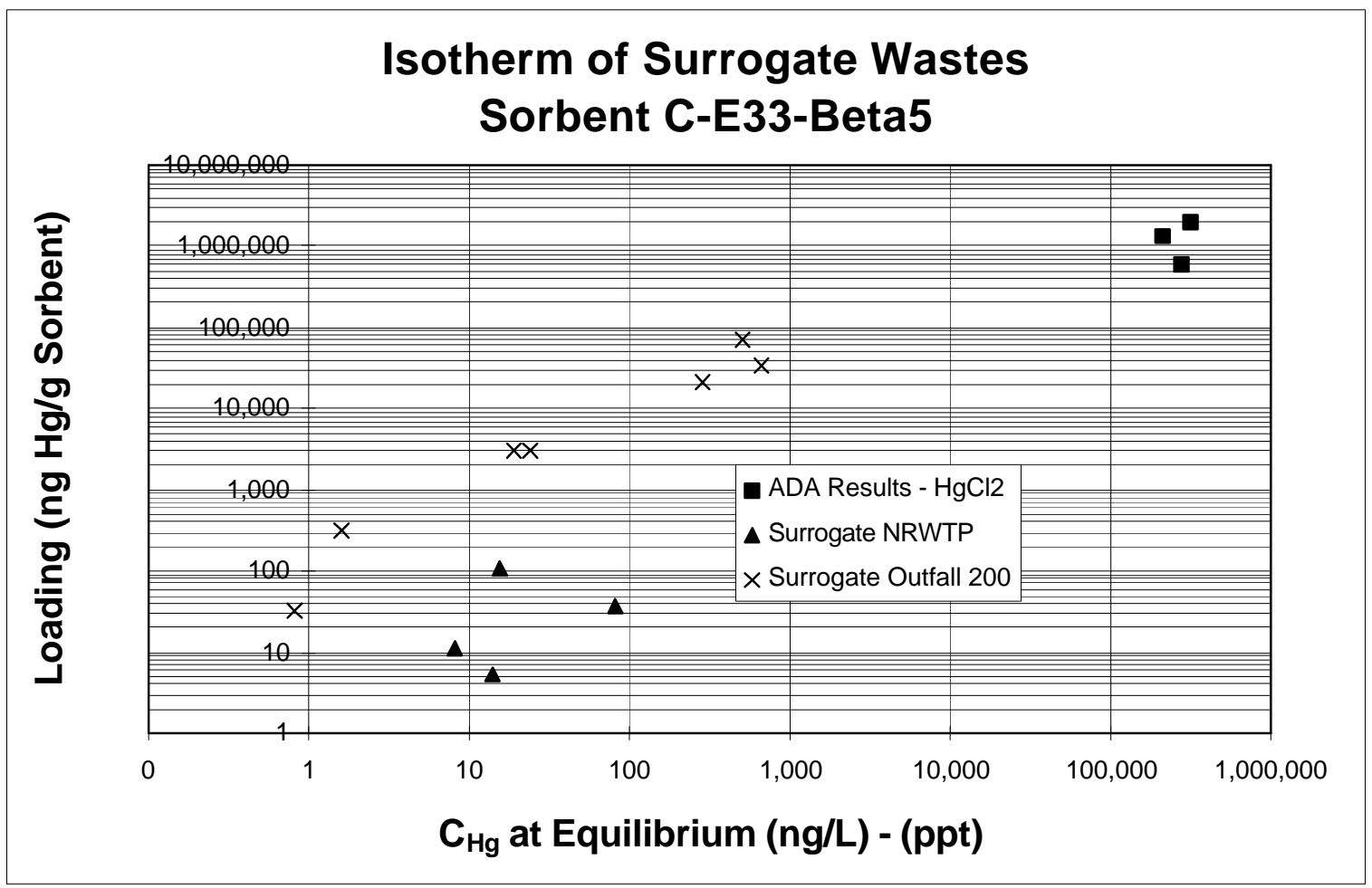

Figure 15 -- Isotherm for C-E33-Beta5 Including Surrogate Results 


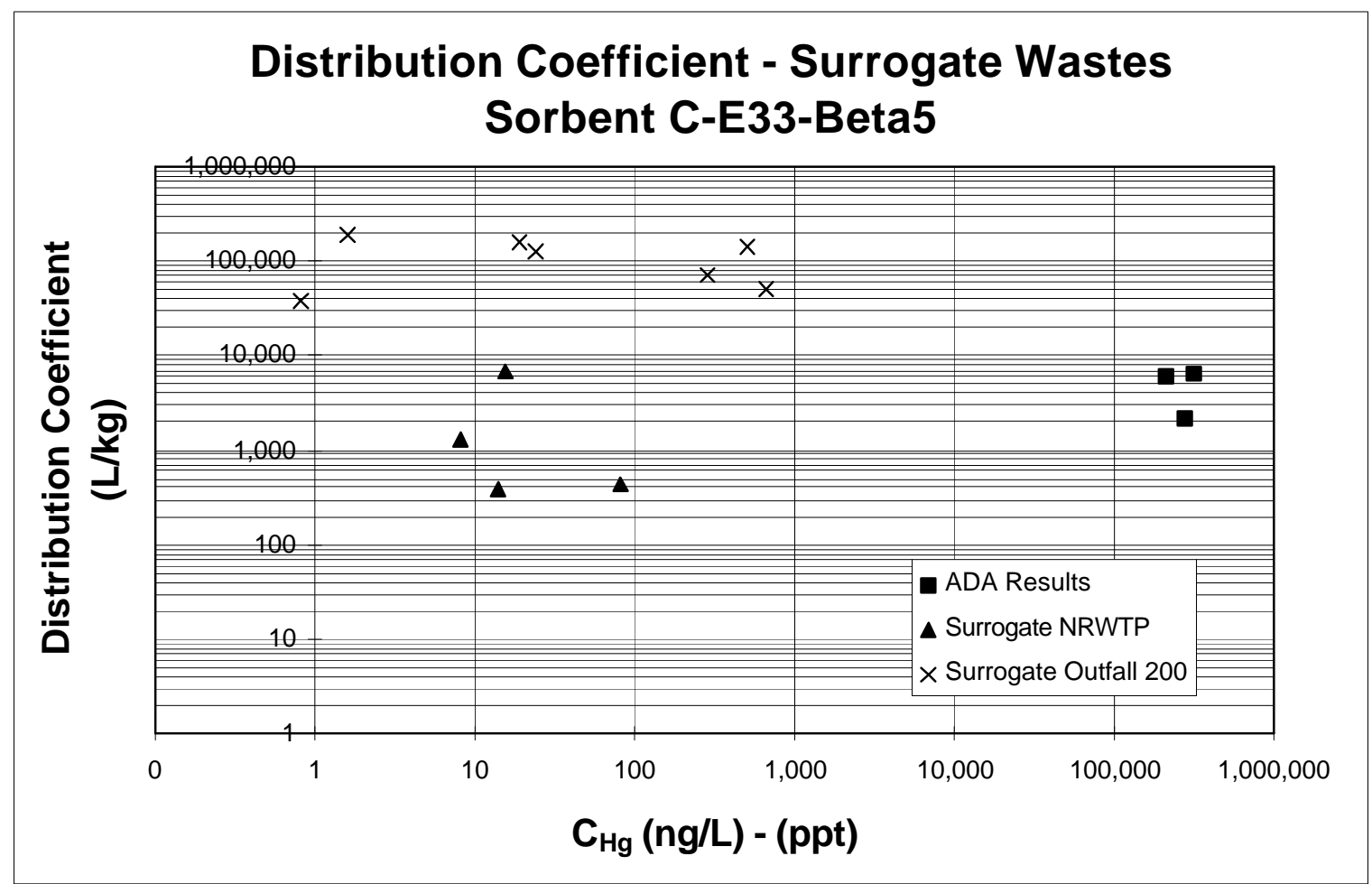

Figure 16 - Distribution Coefficient for C-E33-Beta5 Including Surrogate Results

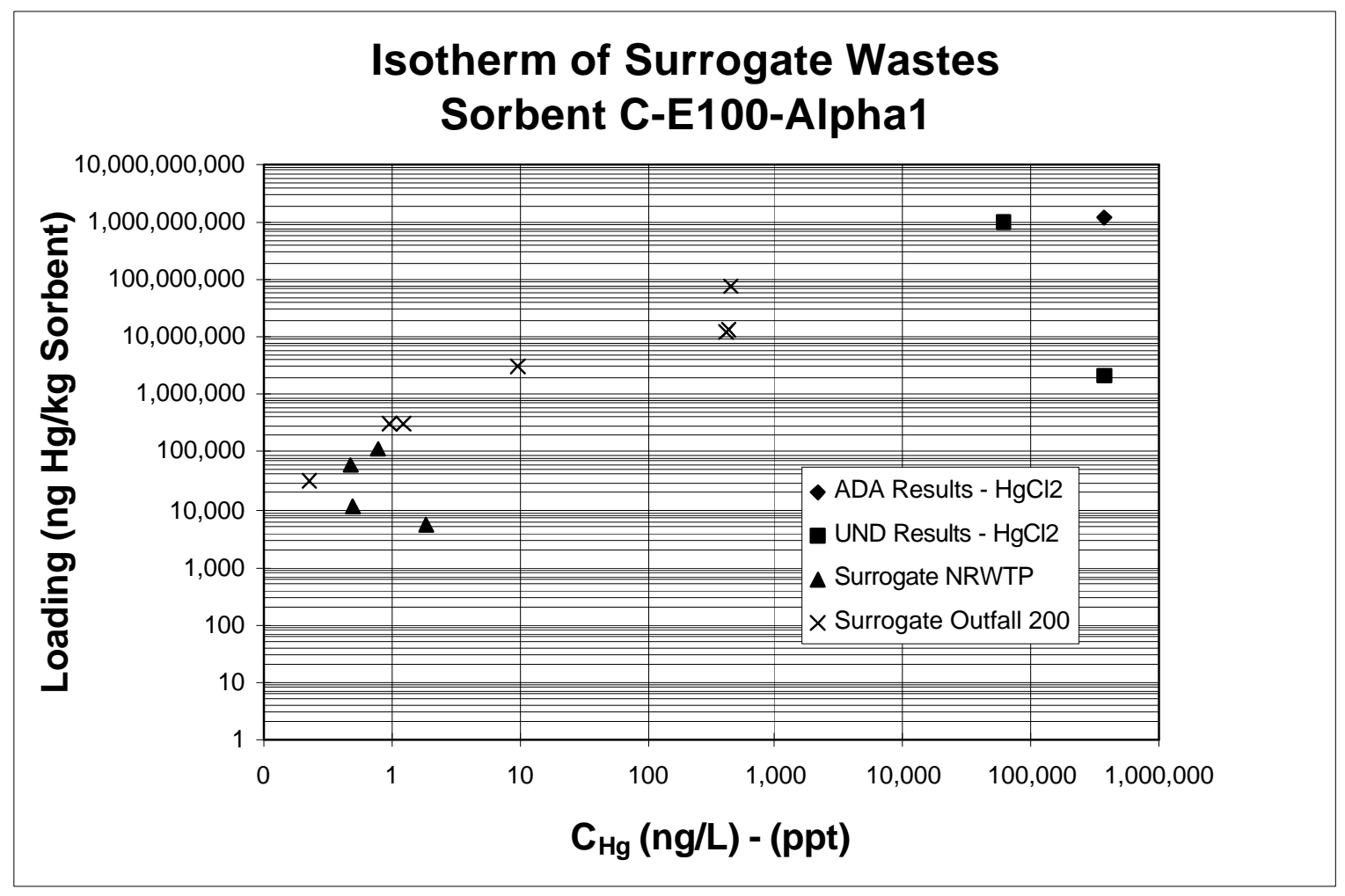

Figure 17 -- Isotherm for C-E100-Alpha1 Including Surrogate Results 


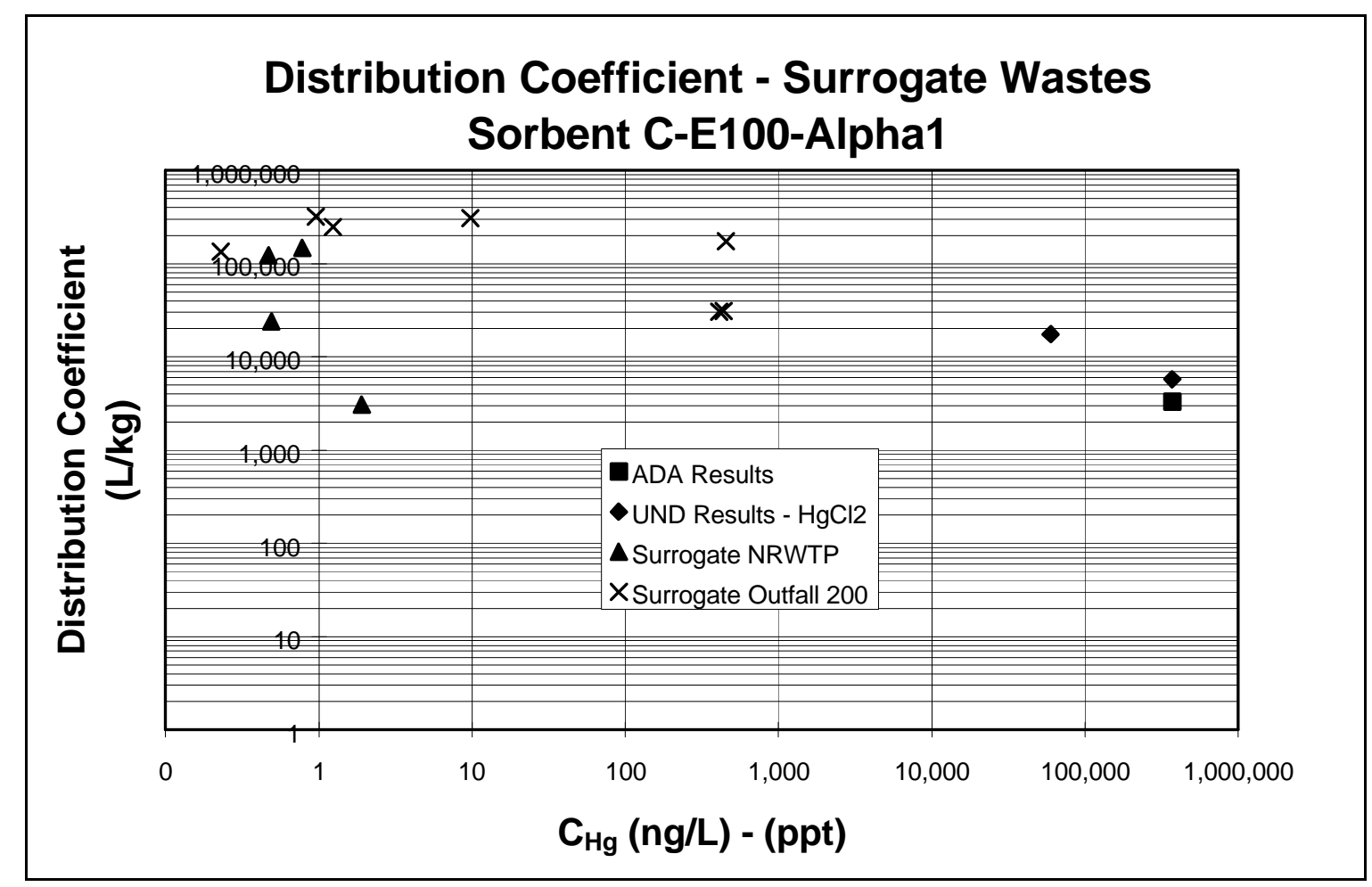

Figure 18 -- Distribution Coefficient for C-E100-Alpha1 Including Surrogate Results

\section{Isotherm Testing - Actual Wastewater}

Isotherm tests using sorbent C-E100-Alpha1 on actual Outfall 200 wastewater were performed in July. ORNL personnel performed these tests, and Frontier Geosciences analyzed the samples. Results are shown in Figure 19. Four samples showed equilibrium levels of mercury below 4 ppt, and one was at 0.24 ppt. The duplicates and spiked samples all looked good, indicating that the series of tests was representative and accurate.

The distribution coefficient is presented in Figure 20. These data show a clear linear trend, although considerable data scatter is seen at lower concentrations. 


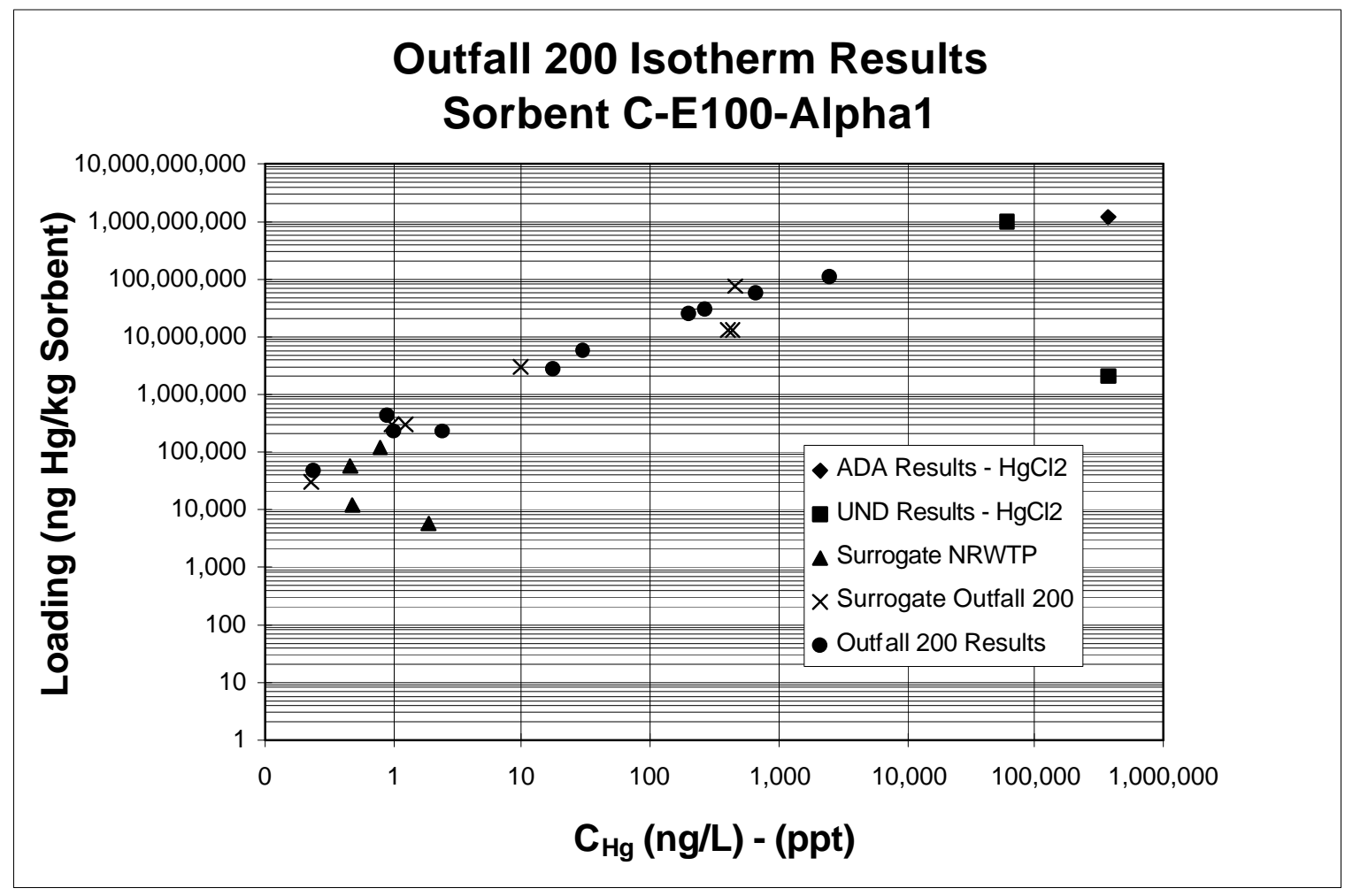

Figure 19 -- Isotherm for Sorbent C-E100-AlphaI Including Outfall 200 Results

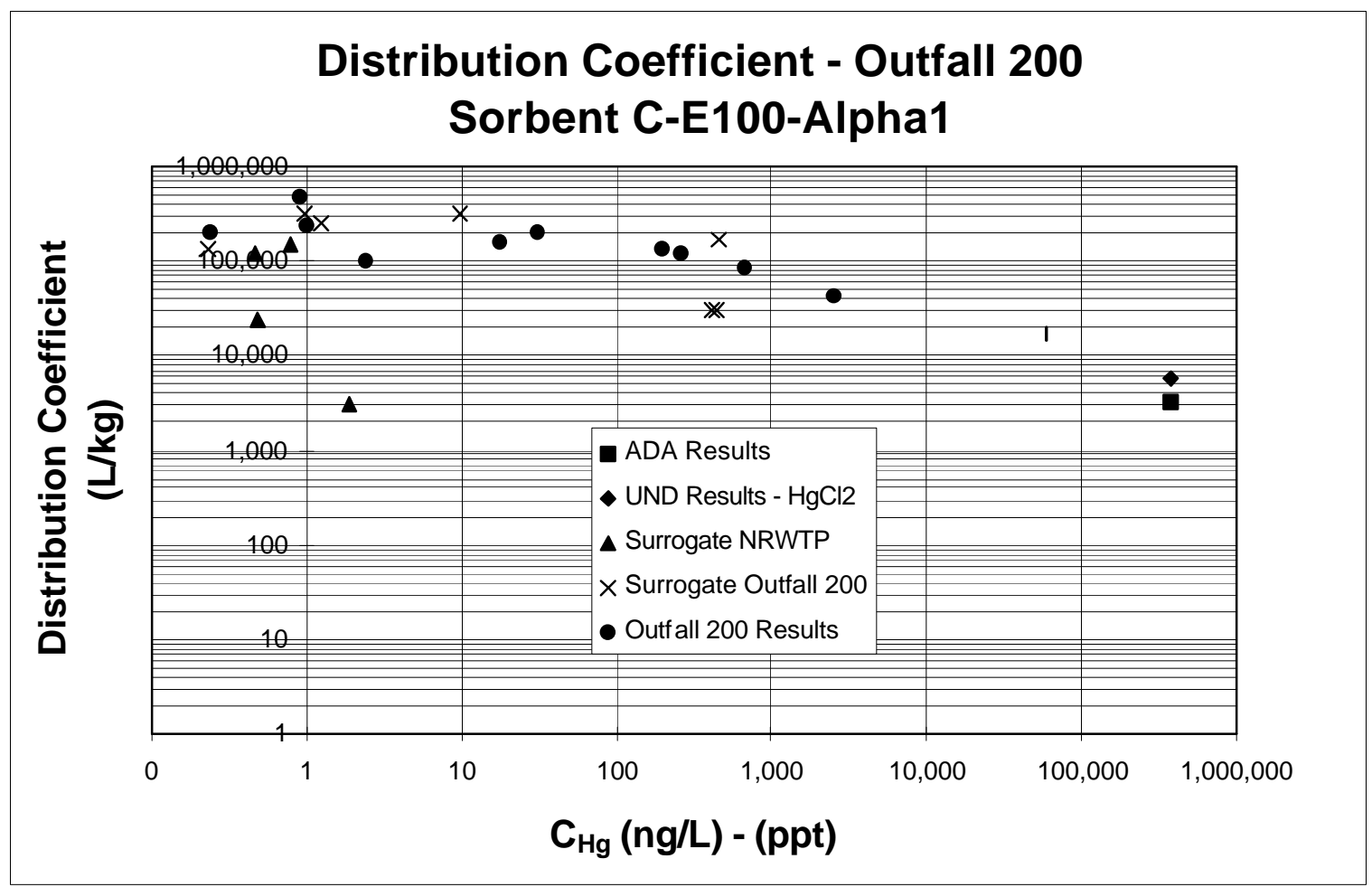

Figure 20 -- Distribution Coefficient for Data in Figure 19 


\section{Thermal Endurance Testing}

A thermogravimetric analysis was performed at UND-EERC on sorbents C-N-X, C-E33-X and C-E100-Alpha1. This analysis consists of weighing a small amount of the sorbent, then heating it in air and measuring the mass loss as the temperature increases. These tests were performed in air, which would supply an oxygen source for combustion of the material. In all cases, the sorbent was thermally stable up to $370^{\circ} \mathrm{C}$, which is the maximum regeneration temperature used by ADA in this process.

To determine if the noble metal crystallites deteriorate with time at regeneration temperature, samples of six sorbents were placed in an oven at $370^{\circ} \mathrm{C}$. This is the "thermal endurance" test. Fourteen one-gram samples of each sorbent were measured into crucibles and placed in the oven. When the samples were checked in September, all of the carbon-based sorbents had turned to ash. The temperature in the oven was checked and it was at $370^{\circ} \mathrm{C}$ as desired. This test indicates that the sorbent is stable in air at elevated temperature for limited periods of time (45 minutes during the TGA), but it is not stable for longer periods. Based on these results, ADA decided to perform regeneration in nitrogen to avoid combustion. 


\section{DEVELOP REGENERATION UNIT}

Key to the cost-effective use of noble metal sorbents for the removal of mercury from process fluid streams (both gas and liquid) is the ability to regenerate and reuse the sorbents multiple times. Past research efforts at ADA have focused on the formulation of sorbent materials to maximize their ability to adsorb mercury and to desorb the bound mercury without measurable long-term degradation of the sorbent. Some success in demonstrating effective mercury removal has led to a renewed emphasis on the development of a practical regeneration process for the sorbents. This is critical, because the final fate of the mercury upon removal from the process stream should be as a non-hazardous waste or recyclable material. When this is accomplished, the mercury is removed from the environment.

The objective of this task was to devise practical methods to regenerate the sorbents. Specific objectives included determining time and temperature required to remove water and mercury from sorbents and identifying methods for capturing the mercury evolved during regeneration. Models for sorption and regeneration were also investigated.

\section{A. Laboratory Regeneration Tests}

UND-EERC performed experiments to determine the most effective regeneration operating parameters. The experiments were performed in air using sorbent C-E100-Alpha1, and results are listed in Table 5. (UND-EERC used air instead of nitrogen because they started the regeneration tests before ADA completed thermal endurance tests). The only measurable loss of mercury occurred in tests 3 and 6; each of these tests was four hours in duration. From this set of tests, it seems that mercury removal is a mass transfer dependent process, not a heat transfer dependent process. To be certain that these values were not due to experimental error, three troubleshooting actions were taken:

- $\quad$ A clean sample of C-E100-Alpha1 sorbent was wetted with deionized water and analyzed to verify the blank value of the sorbent.

- $\quad$ A portion of the wetted clean sorbent was regenerated and analyzed to verify that the test system was not the source of the mercury.

- $\quad$ One of the $300^{\circ} \mathrm{F}, 1-\mathrm{hr}$ tests was repeated with a $\mathrm{KMnO}_{4}$ impinger located at the tube outlet to corroborate the amount of mercury removed with the analytical results obtained during the earlier tests.

Neither the sorbent nor the system was found to be a source of mercury. The impinger corroborated the fact that very little mercury was removed during the test.

These results indicated that a considerably longer residence time at temperature would be required for adequate regeneration of the sorbent to take place. Because ADA had similar results, UNDEERC expanded the experimental matrix to include tests performed for $53 \mathrm{hr}$, with two data 
points performed at $26.5 \mathrm{hr}$. Tests were performed at $300^{\circ} \mathrm{F}$ and $700^{\circ} \mathrm{F}$. To make use of some of the earlier tests, the minimum residence time used during the second matrix was 4 hours. To provide a more complete picture of the regeneration at high temperatures and longer residence times, two additional tests were performed at $700^{\circ} \mathrm{F}$ for 26.5 hours and $800^{\circ} \mathrm{F}$ for 52 hours. All of these tests were performed using the same loaded sorbent as feed and the same regeneration protocol as the earlier runs.

The results of the second set of tests are presented in Table 6. As the table shows, the differences between the $700^{\circ} \mathrm{F}$ and $800^{\circ} \mathrm{F}$ tests performed for the same residence time were not significant. The highest level of regeneration, $99.76 \%$, was attained during the 52 -hour, $700^{\circ} \mathrm{F}$ test. Regeneration at $800^{\circ} \mathrm{F}$ turned the sorbent slightly brown during the test.

Although regeneration at $300^{\circ} \mathrm{F}$ for shorter residence times appeared to remove at least a portion of the mercury, analysis of the regenerated sorbent from the longer $300^{\circ} \mathrm{F}$ tests exhibited an abnormally high mercury level relative to the loaded sorbent and the products of the other regeneration tests. The product of the $300^{\circ} \mathrm{F}, 26.5$-hr test contained more mercury than the product of the $300^{\circ} \mathrm{F}, 53$-hour test, indicating that additional mercury was removed as the residence time increased.

The reason for the high mercury levels in these samples may be due to the fact that the $300^{\circ} \mathrm{F}$ tests were the last ones to be performed and the loaded sorbent had increased in relative mercury concentration through evaporation of some of the water. A more likely explanation is the fact that the total mercury analysis requires a very small quantity of sorbent. If the sorbent is not uniform in surface area or noble metal coating, the bulk sorbent will be heterogeneous enough to give different results for analyses performed on the same sample.

Table 5 -- Results of UND-EERC Regeneration Operating Parameter Matrix

\begin{tabular}{|c|c|c|c|}
\hline Test No. & $\begin{array}{c}\text { Temperature } \\
\left({ }^{(\mathbf{F})}\right.\end{array}$ & $\begin{array}{c}\text { Time at } \\
\text { Temperature (hr) }\end{array}$ & $\begin{array}{c}\text { Mercury Removal } \\
(\mathbf{\%})\end{array}$ \\
\hline 1 & 300 & 1 & 1.82 \\
\hline 2 & 500 & 2.5 & 0.91 \\
\hline 3 & 700 & 4 & 16.00 \\
\hline 4 & 700 & 1 & 1.82 \\
\hline 5 & 500 & 2.5 & -3.64 \\
\hline 6 & 300 & 4 & 14.27 \\
\hline
\end{tabular}


Table 6 -- Results of the Revised Regeneration Operating Parameter Matrix

\begin{tabular}{|c|c|c|c|}
\hline Test No. & $\begin{array}{c}\text { Temperature } \\
\left({ }^{\mathbf{}} \mathbf{F}\right)\end{array}$ & $\begin{array}{c}\text { Time at } \\
\text { Temperature (hr) }\end{array}$ & $\begin{array}{c}\text { Mercury Removal } \\
(\mathbf{\%})\end{array}$ \\
\hline 1 & 300 & 4 & 14.3 \\
\hline 2 & 700 & 4 & 16.0 \\
\hline 3 & 800 & 26.5 & 97.9 \\
\hline 4 & 700 & 52 & 99.8 \\
\hline 5 & 300 & 26.5 & -25.5 \\
\hline 6 & 300 & 52 & -20.9 \\
\hline 7 & 700 & 26.5 & 96.7 \\
\hline 8 & 800 & 52 & 99.0 \\
\hline
\end{tabular}

ADA performed six regeneration tests in nitrogen using sorbent C-E100-Alpha1. In these tests, three samples of the sorbent were weighed out, placed in a 10,000 $\mu \mathrm{g} / \mathrm{l}$ solution of mercuric chloride, and placed on the shaker table for 24 hours (standard isotherm procedure). The three samples were then drained, blotted dry, and placed together in the ADA Mercury Sorption Apparatus. Dry nitrogen was flowed over the sorbent as the temperature in the apparatus was increased to $370^{\circ} \mathrm{C}$. The ADA Continuous Mercury Analyzer was used to monitor the mercury desorbing from the sorbent to determine when all of the mercury was removed.

After regeneration, the sorbent was split into three samples, weighed again, and placed in another $100 \mathrm{ml}$ of mercuric chloride solution. This procedure was repeated three times. Table 7 lists the time and temperature required to regenerate the sorbent. Sorbent C-E100-Alpha1 showed good regeneration characteristics at $370^{\circ} \mathrm{C}$. 
Table 7 -- Regeneration Tests using Mercuric Chloride and Sorbent C-E100-Alpha1

\begin{tabular}{|c|c|c|c|}
\hline Sorbent & $\begin{array}{c}\text { Regeneration } \\
\text { Number }\end{array}$ & $\begin{array}{c}\text { Temperature } \\
\left({ }^{(\mathbf{F})}\right.\end{array}$ & $\begin{array}{c}\text { Time at } \\
\text { Temperature } \\
(\mathbf{h r})\end{array}$ \\
\hline C-E100-Alpha1 & 1 & 700 & 38 \\
\hline C-E100-Alpha1 & 2 & 700 & 48 \\
\hline C-E100-Alpha1 & 3 & 700 & 48 \\
\hline
\end{tabular}

The isotherm results for before and after the regenerations are shown in Figure 21. The sorbent C-E100-Alpha 1 performed very well after regeneration (99.9\% uptake).

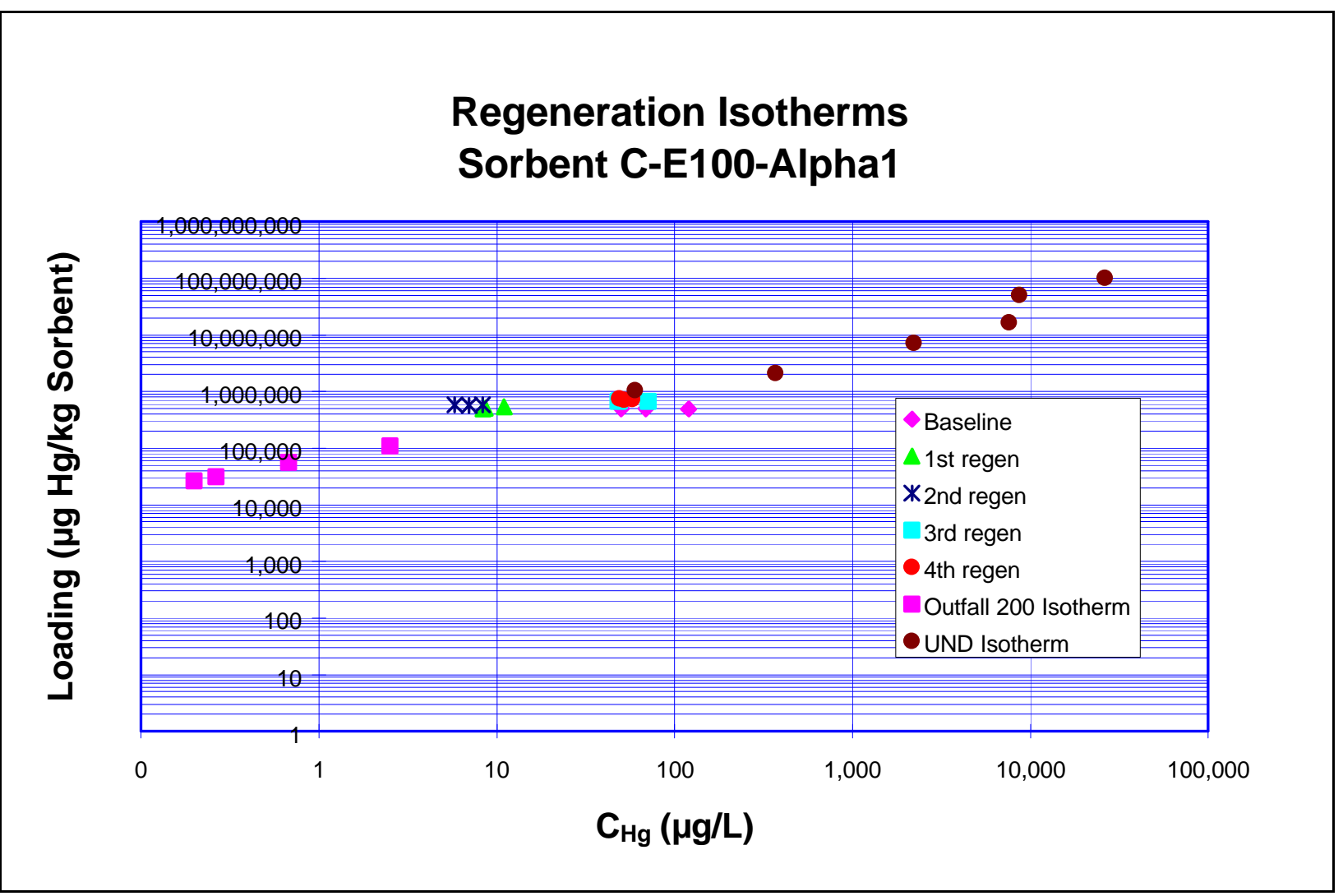

Figure 21 -- Isotherm Results from Regeneration Tests 


\section{B. Mercury Capture Methods for Regeneration Offgas}

One issue in the regeneration of mercury sorbents has been the collection of desorbed mercury in a stable form. To date, the proposed mechanism for this aspect of the process featured condensation of the desorbed material as elemental mercury. Upon closer examination, this mechanism appears to have some problems due to the low concentration of mercury vapor in the purge gas.

Measured mercury concentration in the purge gas from laboratory regeneration experiments has approached one $\mu \mathrm{g} / \mathrm{m}^{3}$, which results in an equilibrium vapor pressure of about $10^{-4}$ torr. The corresponding equilibrium temperature for this pressure is $0^{\circ} \mathrm{C}$; that is, no condensation of mercury will occur until the gas temperature is at or below this equilibrium value. Condensation of $99 \%$ of the mercury vapor at this concentration requires that the gas phase be cooled to below $-38^{\circ} \mathrm{C}$. The mercury vapor concentration in the purge gas discharge from the condenser would then be about $10 \mu \mathrm{g} / \mathrm{m}^{3}$. If purge gas equilibrium mercury concentrations are increased by a factor of 10 , then the temperature required for $99 \%$ condensation only increases to $-25^{\circ} \mathrm{C}$, and the discharge mercury vapor concentration rises to $100 \mu \mathrm{g} / \mathrm{m}^{3}$, which may be of concern.

At such a concentration, an additional removal mechanism such as a sulfur-impregnated activated carbon canister may be required to reduce the mercury concentration in the purge gas before discharge to the atmosphere. There are no current EPA regulations regarding the mercury vapor content of process stream releases to the atmosphere, but the maximum emissions rate for waste to energy plants is $50 \mu \mathrm{g} / \mathrm{m}^{3}$; this would make a reasonable design condition for a regeneration purge gas treatment system.

After the above analysis of condensation for mercury removal from regeneration purge gas was completed, alternatives for stabilization of the mercury in the purge gas were investigated. One promising option was to contact the purge gas with a liquid stabilizing reagent (proprietary). ADA demonstrated in laboratory tests that the liquid reagent is quite reactive with elemental mercury. The liquid reagent reacts with mercury to form a very stable solid compound that has been proven to pass TCLP tests as a stable form of mercury, which means that the reaction product can be disposed of as a non-hazardous waste.

A simple experiment was set up to evaluate the ability of the liquid reagent to remove vapor phase mercury from a simulated purge gas stream. Nitrogen gas was doped with elemental mercury vapor to a concentration of about one $\mathrm{mg} / \mathrm{m}^{3}$. This gas was bubbled through a column of the reagent and the outlet gas stream was analyzed to measure its mercury content. Tests were run at several gas flow rates and at two reagent concentrations. The reagent was shown to be very effective in removing the elemental mercury vapor from the gas stream, reducing the measured concentration to less than $50 \mu \mathrm{g} / \mathrm{m}^{3}$. It appeared from the testing that a key process variable is the gas/liquid contact area; in this experimental setup, large contact area was promoted by generating very small bubbles of simulated purge gas in the reagent column. A sparger was used to generate a large number of very small bubbles, well below one $\mathrm{mm}$ in diameter. These small bubbles provided a very vigorous mixing zone, with recirculation and the formation of a foam floating on the frothy liquid. This contact appeared to be sufficient to remove more than $95 \%$ of 
the vapor phase mercury from the simulated purge gas stream. Results of these tests are shown in Figure 22.

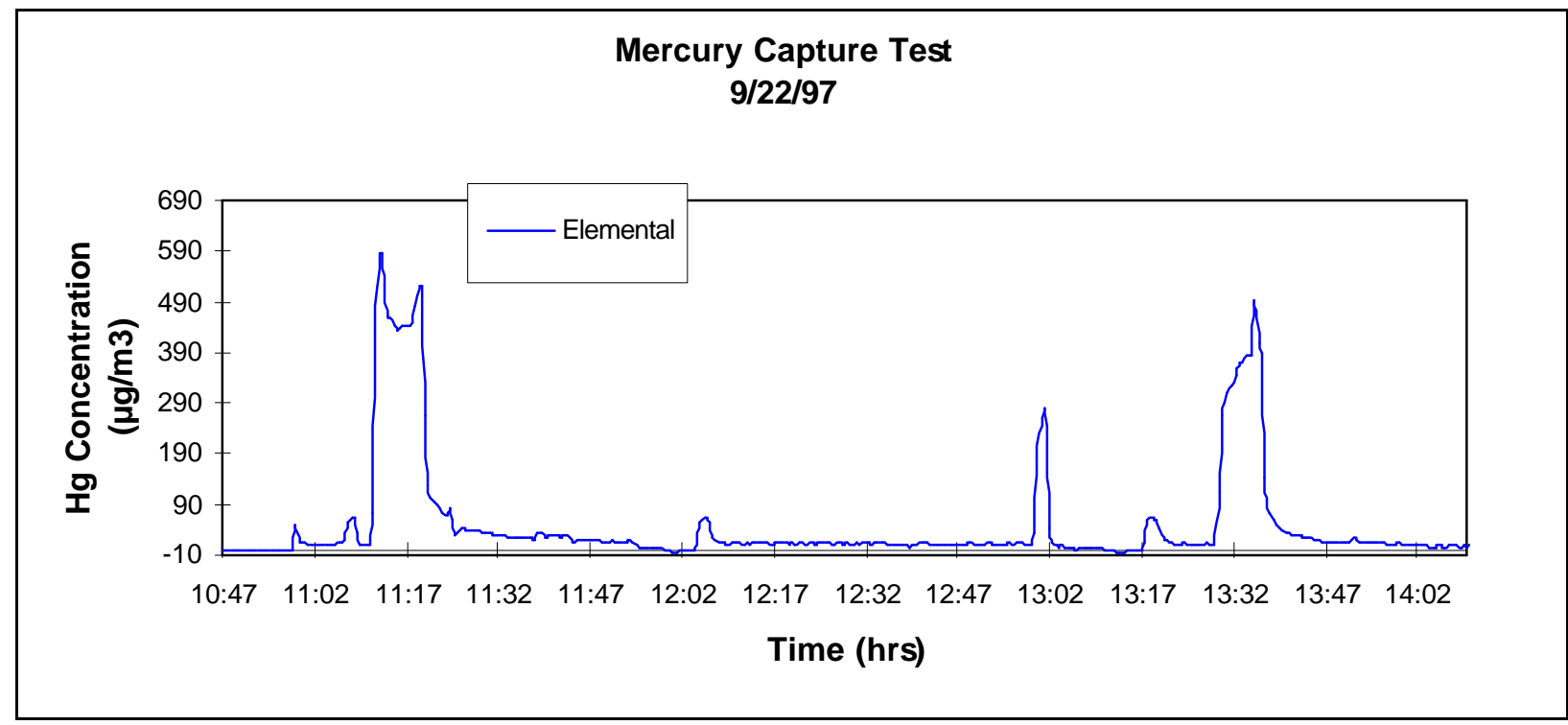

Figure 22 -- Mercury Capture Tests Showing Impact of Scrubber on Vapor Phase Mercury Concentration

Further experimentation will be performed to quantify the removal efficiency of mercury vapor with the reagent. ADA is exploring the design of a regeneration process that incorporates a reactor to remove the mercury from the purge gas via contact with the reagent. This method of mercury stabilization is very attractive in cases where the mercury is from a radioactively contaminated source. The liquid mercury obtained from condensing the offgas would need to be stabilized and disposed of as a low-level radioactive waste. Using the reagent as part of the regeneration unit combines removal with stabilization. The stabilized material can then be filtered from the reagent solution and disposed of.

Another option in mercury capture would be a combination of a condenser and contact with the liquid reagent. If recovery of mercury in an elemental form is required as part of the economics, the majority of the mercury could be condensed, then the gas from the condenser would be run through the reagent to scrub the remainder of the mercury.

An invention disclosure describing this technology has been prepared, and patent proceedings initiated.

\section{Models for Sorption and Regeneration}

Sorption and regeneration models for the treatment system will aid in scale-up of the system. Under the Base Contract, information was compiled for several mass transfer models describing the sorption and desorption process. Modeling these processes will allow ADA to accurately predict 
the amount of water that can be treated using the sorbents and estimate the time required to regenerate the sorbents for continued use.

The first step in developing the model is to derive the generalized material balance. Certain terms in the expression are omitted to simplify the mathematics used to obtain an analytical solution to the problem. In the second step, equations are developed to describe the controlling mass transfer rate. Third, an isotherm is used to describe the equilibrium between the fluid phase and solid (sorbent) phases. The system of equations is then solved using advanced mathematical techniques or numerical methods that give the change of concentration of the solute in the two phases as a function of time and position in a fixed bed. Detailed results from the modeling efforts are provided in Appendix A.

Three models were investigated: Hougen - solid phase, Hougen - liquid phase, and Rosen. Breakthrough curves predicted by the three models for the uptake of mercury from water are shown in Figure 23. As expected the Rosen model predicted longer breakthrough times since it describes the mass transfer rate for both fluid and intraparticle diffusion.

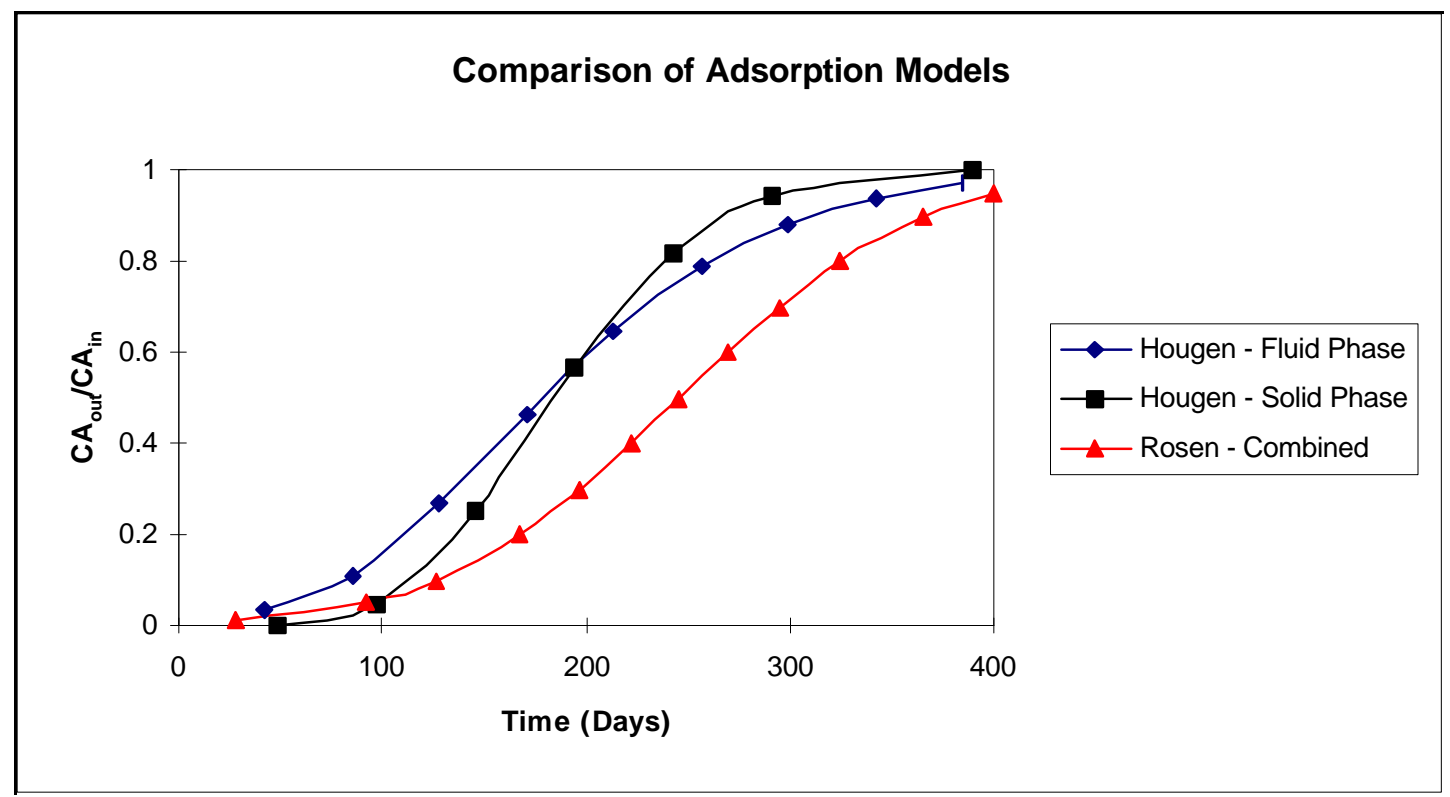

Figure 23 -- Comparison of Adsorption Models 


\section{FIELD TEST OF SORPTION PROCESS}

The objective of this task was to devise engineering scale-up rules for the sorption unit operations and to determine viability of the process under field conditions. The sorbent used in the field tests was C-E100-Alpha1 mixed with a low cost, minimally treated sorbent. The purpose of mixing the sorbents was to reduce treatment costs. Both sorbents were tested with the surrogate wastes, so ADA had a high degree of confidence that the sorbent mix was capable of reducing mercury concentrations to less than $1 \mathrm{ppt}$.

A design for the field-scale sorption-regeneration unit was developed at UND-EERC. It should be noted that this design was performed without the benefit of any laboratory data concerning either regeneration or larger-scale adsorption at low mercury concentrations. The design was based on appropriate rules of thumb for adsorber design as well as pressure drop calculations. It was checked using the data from the ultra-low concentration isotherm tests and found to be appropriate in size.

Three sorbent beds are used during the field tests (Figure 24). Two beds are used in series at all times. This allows for the third bed to be undergoing regeneration without interruption of the treatment process.

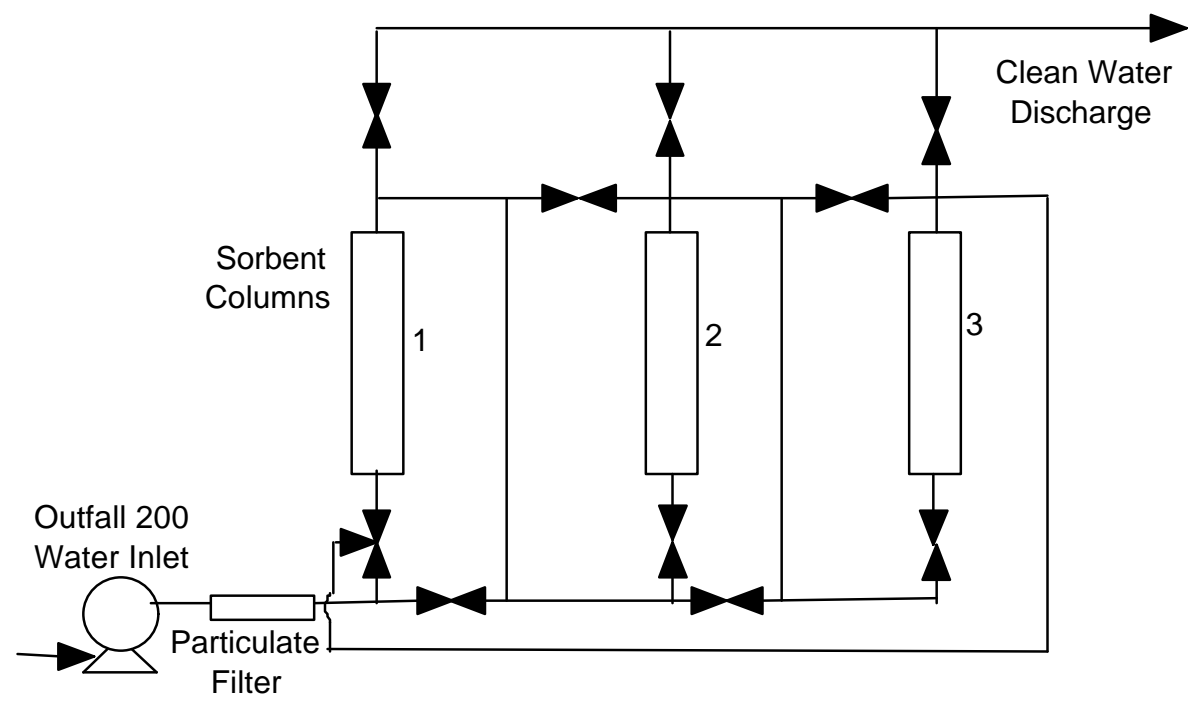

Figure 24 -- Diagram of Mercury Sorption Unit

The sorbent columns are 28 -inch long stainless steel tubes with a 4 -inch inner diameter. The sorbent bed depth is 24 inches. A diaphragm pump draws the water from the stream, through a 1micron filter and into the sorption unit. Outfall 200 water flows upwards through the sorbent beds. Figure 25 shows the valve arrangements at the inlet and outlet of each sorbent column. These valves allow the operator to switch the flow of liquid through different beds. When the first column is showing breakthrough of mercury above acceptable levels, the flow will be switched so that the second and third columns treat the water while the first column is being regenerated. 
Before any contaminated water was passed through the sorption unit, clean water was sent through the unit to ensure leak-free operation.

Fabrication of the field-scale sorption-regeneration unit was completed in August. The unit is on a 4' X 8' platform and contains a regeneration station. Initially, regeneration will take place in a laboratory at ORNL to document and closely monitor the process. Four stainless steel columns that hold the sorbent were designed and built for the field tests. Three columns are available for sorption at any one time, although only two columns are used in series in the field tests. The columns were pressure tested to 300 psi with water and no leaks were detected.

During August, a $9 \times 12 \mathrm{ft}$ portable enclosure was set up on the east bank of the creek near the outlet of Outfall 200. This is the enclosure for the sorption skid. Within the enclosure was set up the pump, PTFE particulate filter and inlet line that is used for the field tests. The inlet of the PTFE line feeding the pump was secured to the metal grid at Outfall 200. Water from Outfall 200 was pumped up to the enclosure, and then back into the creek. The purpose of this step was to ensure that water can be delivered to the sorption unit without any difficulty and that the lines, particulate filter, and wetted pump parts are conditioned with the mercury present in the water. The lines and pump came to equilibrium within a few days.

The sorption skid was transported from UND-EERC on September 2 and arrived at ORNL on September 3. The skid was unloaded on September 4, and final assembly was done that day. A photograph of the unit at Outfall 200 is provided in Figure 25.

Figure 26 shows the results of the field testing. This figure shows the concentrations at the inlet of the 1-micron filter, outlet of the filter (inlet to column 1), outlet of column 1, outlet of column 2, and outlet of the whole skid. The outlet of the skid should give the same mercury value as the outlet of column 2 . After leak checking the system on September 4, water was allowed to flow through the empty columns overnight. Samples were taken on September 5 at five points to determine if mercury was being lost on the walls of the columns and tubing. These are the initial data points on the figure. Two kilograms of sorbent was then added to each of the three columns. The sorbent was allowed to degas over the weekend by flowing water into each of the columns, followed by rapping the sides to remove the air bubbles. This process was continued over the weekend to remove all of the trapped air.

Actual testing of Outfall 200 water started September 8, 1997. The initial flowrate of mercury through the columns was $500 \mathrm{cc} / \mathrm{min}$. Samples were taken every day for the first week then every other day the second week. Twice-a-week sampling started the third week and continued for the duration of the tests. Initially, the field unit removed mercury from Outfall 200 to $1 \mathrm{ppt}$ from a starting concentration of approximately 1,000 ppt. The treatment objective is to remove mercury to below $50 \mathrm{ppt}$. After eight months of testing, the outlet concentration is still below $50 \mathrm{ppt}$. 


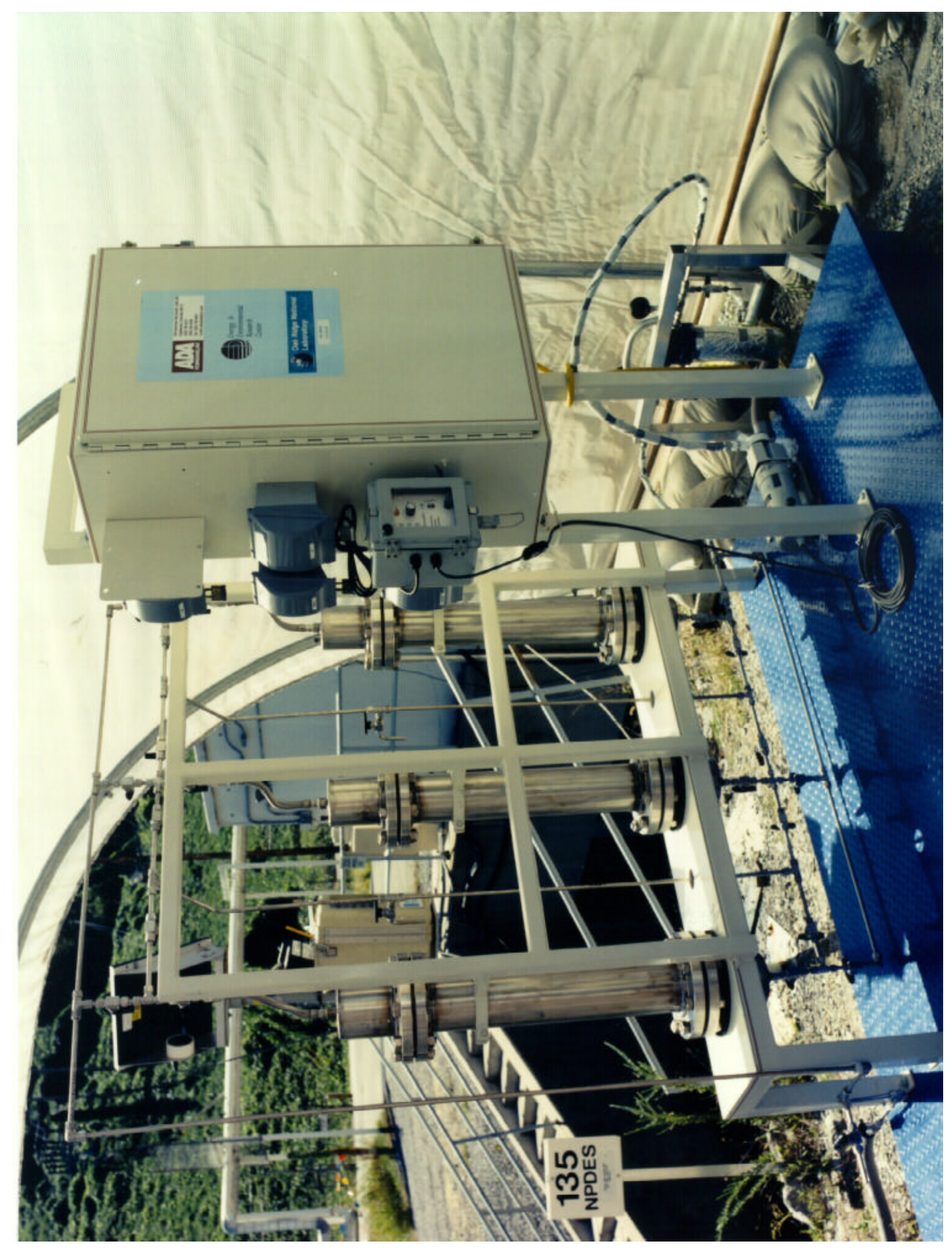

Figure 25 -- Pilot-Scale Mercury Sorption Skid at Outfall 200 


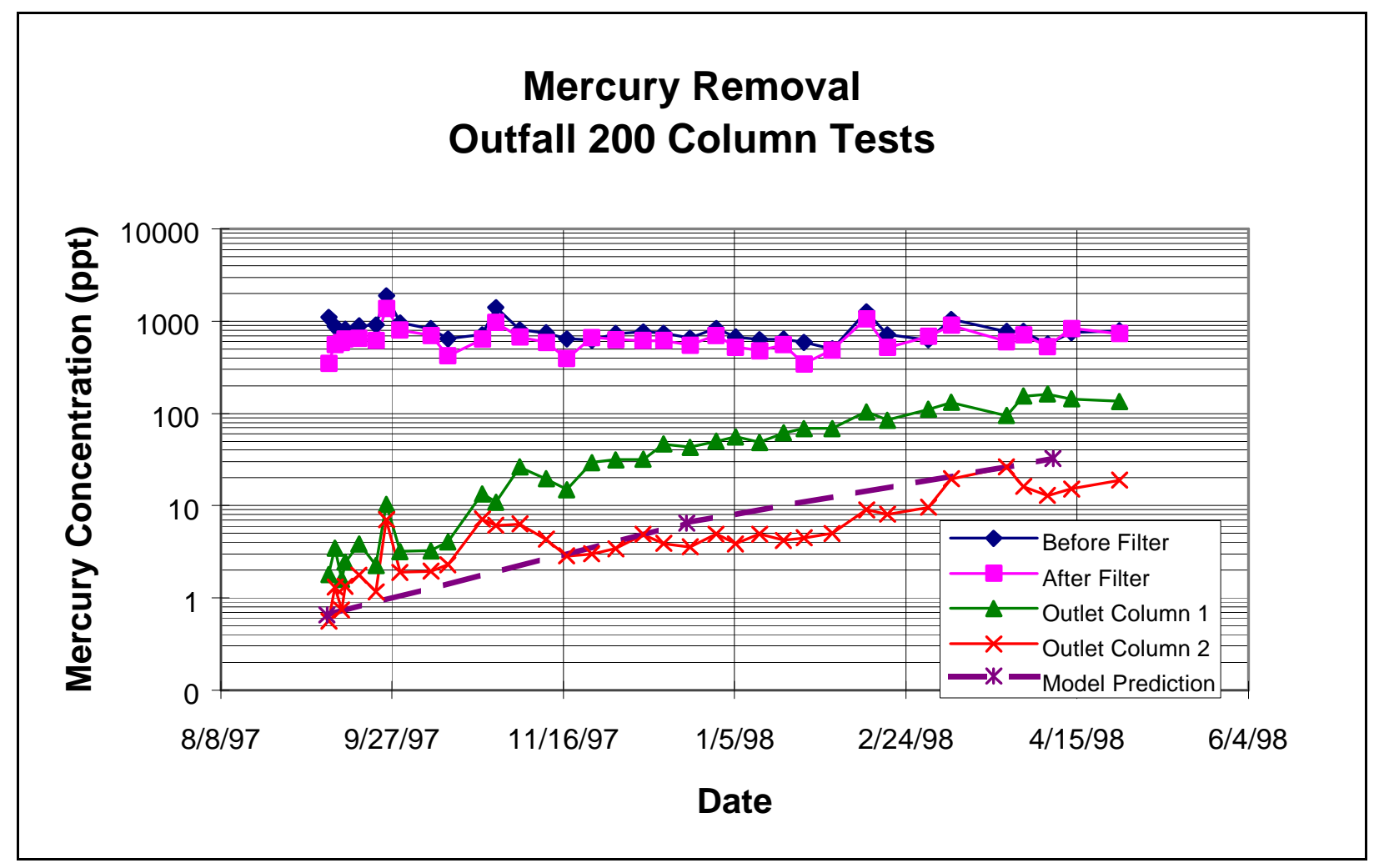

Figure 26 -- Results from Field Test at Outfall 200 


\section{CONCLUSIONS}

This program emphasized the making and testing of superior sorbents for mercury removal, testing of the sorption process at a DOE site, and determining the regeneration conditions in the laboratory. During this project, ADA demonstrated the following key elements of a successful sorption process:

- Sorbents that have a high capacity for dissolved, ionic mercury

- Removal of ionic mercury at greater than $99 \%$ efficiency

- Thermal regeneration of the spent sorbent

ADA synthesized seven sorbents in duplicate batches and tested them with mercuric chloride solutions. The best three sorbents were then tested on surrogate waste containing mercury, and the best sorbent was tested on actual DOE wastewater from the NRWTP and Outfall 200 at the Y-12 Plant in Oak Ridge, Tennessee.

One of the objectives of this program was to demonstrate the ability to manufacture sorbents with uniform qualities. Duplicate isotherm batches showed reproducibility at batch sizes of near $1 \mathrm{~kg}$. Reproducibility of results between laboratories was also demonstrated. In addition, preparation of four $10-\mathrm{kg}$ batch was successfully completed. Laboratory tests showed that these larger batches compared favorably with the smaller batches.

ADA and its subcontractors tested the seven sorbents in duplicate batches using mercuric chloride solutions. At high mercury concentrations, the sorbents take up more than ten percent of the noble metal weight in mercury. All of the sorbents exhibited excellent mercury removal.

Three of the best sorbents were then tested on two surrogate wastewaters from ORNL. Isotherm tests on surrogate Outfall 200 showed greater than $99.9 \%$ removal with two of the sorbents, resulting in a final mercury concentration of less than $1 \mathrm{ppt}$. Mercury concentration in surrogate NRWTP water was reduced to $0.5 \mathrm{ppt}$ in several samples from a starting concentration of 210 ppt.

Laboratory- and pilot-scale tests on actual Outfall 200 wastes were also successful. Isotherms using one sorbent (C-E100-Alpha1) showed mercury removal to below 1 ppt from a starting concentration of 1,000 ppt. The treatment objective was 50 ppt.

Sorption and regeneration models for the treatment system showed good correlation with the field data. The models predict a breakthrough time of four months assuming a 10-ppt treatment objective and about seven months for a treatment objective of $50 \mathrm{ppt}$. Actual data from the sorption unit show $10 \mathrm{ppt}$ discharge concentration at six months.

A key element to the cost-effective use of noble metal sorbents for removing mercury from liquid waste streams is the ability to regenerate and reuse the sorbents multiple times. Laboratory-scale regeneration tests demonstrated that regeneration is feasible at $700^{\circ} \mathrm{F}$ over 52 hours in an inert atmosphere. Sorption behavior is not affected after four cycles. 


\section{FUTURE WORK}

The next testing phase (Option 1) will focus on the following objectives:

- Optimize the sorbent configuration. Develop and test sorbent configurations that are less expensive and/or have higher mass transfer rates than the sorbents tested in the base phase

- Design, construct, and test a pilot-scale regeneration unit. Determine field regeneration conditions using equipment that can be scaled up. Demonstrate the field prototype unit at a DOE site

- Determine process economics for cradle-to-grave treatment of mercury from wastewater

Optimizing the sorbent configuration will concentrate on two areas. First, improving mass transfer of mercury to the sorbent will result in higher flow through the sorbent bed. Second, ADA will investigate ways to reduce the cost of the sorption system. For example, using a dualbed system with an inexpensive activated carbon bed at the front end and the more expensive specialty sorbent as a polishing step will reduce costs without compromising performance.

ADA will design, construct, and test a pilot-scale regeneration unit using equipment that can be scaled up. The regeneration unit will consist of the dryer/calciner and the scrubber system for removing mercury from the offgas. The ultimate goal is stabilized mercury ready for disposal as a non-hazardous waste. The unit will be tested at ADA before shipment to ORNL for testing at Outfall 200.

ADA will also continue sampling from the existing mini-pilot unit at Outfall 200 until mercury concentrations of $50 \mathrm{ppt}$ are reached at the outlet of the second column, indicating that breakthrough has occurred. At this time, the first column in the series will be taken off-line for regeneration. The second and third columns will then be put back in operation. The optimized sorbent will be placed in the spare columns on the skid and tested with actual Outfall 200 water at varying flow rates. These tests will be performed in parallel with the existing two-column system.

Finally, ADA will develop detailed economics for a full-scale system. The full-scale design will be based on sorption, regeneration, capture, and disposal of mercury from Outfall 200. The economics will include a comparison with another sorbent that can achieve the same mercury levels in the effluent (less than $10 \mathrm{ppt}$ ). 


\section{APPENDIX A. MODELS FOR SORPTION AND REGENERATION}

\section{General Mass Balance Equation}

Adsorption or desorption of a granular fixed-bed is characterized by its unsteady state nature with the concentration of the solute being dependent on the bed position $(\mathrm{Z})$ and with respect to time (t). Using the shell balance method, a material balance of the system can be derived. The differential mass balance for a fixed-bed adsorber and the accompanying assumptions are presented below.

$$
\left(D_{a a} \frac{\partial^{2} C_{A}}{\partial Z^{2}}\right)_{t}-\left(U_{Z} \frac{\partial C_{A}}{\partial Z}\right)_{t}=\left(\frac{\partial C_{A}}{\partial t}\right)_{z}+\left(\frac{(1-\varepsilon)}{\varepsilon} \frac{\partial C_{A s}}{\partial t}\right)_{Z}
$$

Where: $\quad D_{a a} \quad$ axial dispersion diffusivity coefficient for component A

$U_{z} \quad$ interstitial velocity of the fluid through the bed

$\varepsilon \quad$ porosity of the bed (void fraction)

$C_{A} \quad$ molar concentration of component $\mathrm{A}$ in the fluid phase

$C_{A s} \quad$ molar concentration of component $\mathrm{A}$ in the solid phase

Z axial dimension

$t \quad$ time dimension

\section{Mass Balance Assumptions}

1) Axial diffusion coefficient is constant with respect to the concentration

2) Velocity of the fluid through the bed is constant

3) Adsorption operation is nearly isothermal since the concentration of adsorbate (mercury compounds) is sufficiently dilute and due to the high heat capacity of water. (This assumption eliminates the need for an energy balance.)

4) Fluid moves through the bed with a plug flow velocity profile (This assumption eliminates momentum balance.)

5) No radial concentration gradient exists at a given axial position in the bed.

The adsorption of material by a sorbent is a rate process that can be controlled by the external mass transfer rate from the fluid stream to the solid, an internal mass transfer rate as a component is adsorbed onto a sorbent surface and moves through the pores of the particle, or a combination of both mechanisms. Breakthrough curves for each of these cases were determined.

\section{The Hougen - Marshall Model}

The Hougen-Marshall model was used to model the uptake of elemental mercury from water for the cases where external mass transfer through the fluid phase to the sorbent controls the rate of adsorption and for when internal mass transfer through the particle controls the rate of 
adsorption. This model was proposed in 1947 for modeling the recovery of solvent vapors by activated carbon, drying of gases by silica gel and the clarification of liquids by charcoal.

In their formulation, the general mass balance equation was simplified by neglecting the axial diffusion term as well as the change of fluid concentration with respect to time. A linear isotherm was used to provide the equilibrium relationship between the concentration of solute in the fluid and solid phases. What resulted was a pair of differential equations describing the concentration change of component $\mathrm{A}$ in the fluid and solid phases as a function of time and position in the bed.

$$
\begin{aligned}
& \frac{\partial \bar{Q}}{\partial \tau}=\bar{X}-\bar{Q} \\
& \frac{\partial \bar{X}}{\partial \zeta}=\bar{Q}-\bar{X}
\end{aligned}
$$

The parameters $Q, \bar{X}, \tau$, and $\zeta$ are dimensionless variables used to simplify the mathematics, where:

$$
\begin{aligned}
& \bar{X}=\frac{C_{A}}{C_{A o}} \\
& \bar{Q}=\frac{q_{A}}{q_{A}^{\infty}}
\end{aligned}
$$

With the following boundary conditions:

$$
\begin{aligned}
& \mathrm{C}_{\mathrm{A}}=\mathrm{C}_{\mathrm{A} 0} \text { at } \bar{X}=1 \text { at } \zeta=0 \text { for all } \tau \\
& \mathrm{q}_{\mathrm{A}}=0 \text { at } Q=0 \text { at } \tau=0 \text { for all } \zeta
\end{aligned}
$$

Here: $\quad C_{A} \quad$ fluid phase concentration of component $\mathrm{A}, \mathrm{kgmoles} / \mathrm{m}^{3}$

$C_{A 0} \quad$ initial concentration of component A entering the adsorber, kgmoles $/ \mathrm{m}^{3}$

$q_{A} \quad$ amount of component $\mathrm{A}$ on the solid phase, kgmoles/grams sorbent

$q_{A}^{\infty} \quad$ saturation capacity of component A on the solid phase, kgmoles/grams

sorbent

The variables $\tau$ and $\zeta$ are called the bed length and time parameters, respectively and will be defined later for each of the two mass transfer rate cases. The analytical solutions to this set of differential equations is given by equations (6) and (7).

$$
\begin{aligned}
& \bar{X}=1-\int_{0}^{\tau} e^{-(\tau+\zeta)} I_{o}(\sqrt{4 \zeta \tau}) d \tau \\
& \bar{Q}=\int_{0}^{\zeta} e^{-(\tau+\zeta)} I_{o}(\sqrt{4 \zeta \tau}) d \zeta
\end{aligned}
$$




\section{External Mass Transfer Rate Controlling}

For the case in which the adsorption process is controlled by the rate of mass transfer through the fluid boundary layer surrounding the sorbent particles, the parameters $\tau$ and $\zeta$ are defined by equations (8) and (9).

$$
\begin{aligned}
& \zeta=\frac{Z k_{f} a}{\varepsilon U_{Z}} \\
& \tau=\frac{k_{f} a}{K_{D} \rho_{B}}\left(t-\frac{Z}{U_{Z}}\right)
\end{aligned}
$$

Here: $\quad k_{f} \quad$ fluid phase mass transfer coefficient, $\mathrm{m} / \mathrm{s}$

$a \quad$ interfacial area of bed per volume of bed, $\mathrm{m}^{2} / \mathrm{m}^{3}$

$K_{D} \quad$ distribution coefficient, liters $/ \mathrm{kg}$ of sorbent

$\rho_{B} \quad$ bulk density of the sorbent, $\mathrm{kg} / \mathrm{m}^{3}$

The fluid mass transfer coefficient was calculated from a correlation developed by Sherwood for liquids and gases in packed beds of spherical or cylindrical particles.

$$
\begin{aligned}
& k_{f}=\frac{D_{A B}}{d_{p}}(1.17) \operatorname{Re}^{0.585} S c^{1 / 3} \\
& \operatorname{Re}=\frac{d_{p} \mathrm{\rho}_{l} U_{s}}{\mu} \quad \text { and } \quad S c=\frac{\mu}{\rho_{l} D_{A B}}
\end{aligned}
$$

Where: $\quad k_{f} \quad$ mass transfer coefficient, $\mathrm{m} / \mathrm{s}$

$d_{p} \quad$ sorbent particle diameter, $\mathrm{m}$

$\rho_{l} \quad$ liquid density, $\mathrm{kg} / \mathrm{m}^{3}$

$U_{s} \quad$ liquid superficial velocity, $\mathrm{m} / \mathrm{s}$

$\mu \quad$ liquid viscosity, cPoise

$D_{L} \quad$ liquid diffusivity coefficient for mercury in water, $\mathrm{m}^{2} / \mathrm{s}$

A general equation was used for predicting the liquid diffusivity of mercury in water. The equation proposed by Sitaraman, Ibrahim and Kuloor (1963) is applicable when water is the solvent. 


$$
D_{L}=16.79 \times 10^{-14}\left(\frac{M_{B}^{1 / 2} \Delta H_{B}^{1 / 3} T}{\mu_{B} V_{A}^{1 / 2} \Delta H_{A}^{0.3}}\right)^{0.93}
$$

Where: $\quad D_{L} \quad$ diffusion coefficient, $\mathrm{m}^{2} / \mathrm{s}$

$M_{B} \quad$ molecular weight of the Solvent, $\mathrm{kg} / \mathrm{kgmole}$

$\Delta H_{B} \quad$ latent heat of vaporization of the solvent at the normal boiling point, joules $/ \mathrm{kg}$

$\mu_{B} \quad$ viscosity of the solvent, cPoise

$\Delta H_{A} \quad$ latent heat of vaporization of the solute at the normal boiling point, joules $/ \mathrm{kg}$

$V_{A} \quad$ molar volume of the solute at the normal boiling point, $\mathrm{m}^{3} / \mathrm{kgmole}$

$T$ absolute liquid temperature, ${ }^{\circ} \mathrm{K}$

At $298^{\circ} \mathrm{K}$, the diffusion coefficient for mercury in water was estimated to be $2.8 \times 10^{-9} \mathrm{~m}^{2} / \mathrm{s}$. A second estimate of the diffusion coefficient was made using the Wilke-Chang equation for dilute solutions of nonelectrolytes. The value of the diffusion coefficient from the Wilke-Chang relation was $3.2 \times 10^{-9} \mathrm{~m}^{2} / \mathrm{s}$.

The mass transfer coefficient, $\mathrm{k}_{\mathrm{f}}$, was calculated based on a water treatment rate of 30 liters/hour at $25^{\circ} \mathrm{C}$. The column used for the treatment is 2 foot long with a 4 inch diameter, capable of holding $2 \mathrm{~kg}$ of sorbent. The sorbent was taken to be $1 / 8$ " diameter spherical beads with a bed porosity of $47 \%$. Using the calculated diffusion coefficient of $2.8 \times 10^{-9} \mathrm{~m}^{2} / \mathrm{s}$, the fluid phase mass transfer coefficient was determined to be $1.5 \times 10^{-5} \mathrm{~m} / \mathrm{s}$.

The sorbent surface area available for mass transfer is expressed on a per bed volume basis and designated by the $\operatorname{symbol} a$. Actual data on this parameter was available from the sorbent vendor. Instead, it was calculated to be $1 \times 10^{3} \mathrm{~m}^{2} / \mathrm{m}^{3}$ using the equation, $a=\frac{6(1-\varepsilon)}{d_{p}}$.

A value for the distribution coefficient $\left(\mathrm{K}_{\mathrm{D}}\right)$ was determined from isotherm data contained in this report. The data followed a Fruendlich isotherm having a Fruendlich capacity constant (K) equal to $173,000(\mathrm{ng} / \mathrm{kg})(\text { liters } / \mathrm{ng})^{0.85}$ and an isotherm intensity constant $(1 / \mathrm{n})$ of 0.85 . By relating the sorbent capacities for the Fruendlich and linear isotherm equations at a mercuric chloride concentration of $1000 \mathrm{ng} / \mathrm{liter}, \mathrm{K}_{\mathrm{D}}$ was calculate to be 63,000 liters $/ \mathrm{kg}$ of sorbent.

The breakthrough curve for a 2 foot adsorption column was determined by integrating equation (6) from zero to $\zeta$ for different values of $\tau$. Integration was accomplished using the simple trapezoid method. $\bar{X}$ values determined by this method were compared to the graphical solution presented by Vermeulen, Klein and Hiester (1973) which showed the two methods gaves similar values. The value of the bed length parameter $(\zeta)$ used in the integration was 8.8 as calculated from equation (8). Estimated time for the $10 \%, 50 \%$, and $90 \%$ breakthrough was 80,165 , and 295 days, respectively. 


\section{Internal Mass Transfer Rate Controlling}

Equations (6) and (7) can also be used to determine the breakthrough curve of an adsoption operation for the case when the process is controlled by the mass transfer rate of adsorbed molecules within the sorbent particles. The mass transfer rate within the sorbent particle has been described by two mechanisms; pore diffusion and surface diffusion. The first mechanism is when the adsorbed component moves through the particle in the fluid contained in the pores of the sorbent. The second mechanism is characterized by the movement of the adsorbed molecule along the wall of the pore structure. A comparison of the two mechanisms for organic compounds has shown that surface diffusion flux is typically one to two orders of magnitudes larger than that in the bulk of the pores [Hand et al. (1983) and Chatzopoulos et al. (1995)]. To apply the Hougen-Marshall model to this case, the dimensionless variables $\tau$ and $\zeta$ are redefined by equations (14) and (15). Definitions for $Q$ and $\bar{X}$ are the same for either case and are given in equations (4) and (5).

$$
\begin{gathered}
\zeta=\frac{Z k_{S} a K_{D} \rho_{B}}{\varepsilon U_{Z}} \\
\tau=k_{s} a\left(t-\frac{Z}{U_{Z}}\right)
\end{gathered}
$$

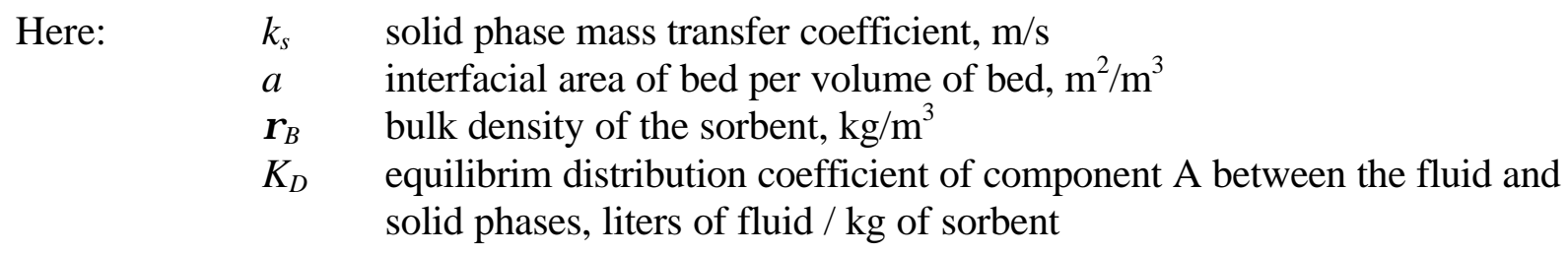

Surface diffusivities for elemental or speciated mercury compounds were not known at the time of our work. However, a procedure for estimating the surface diffusivities was found in the literature [Hand et al. (1983)]. In lieu of actual surface diffusion data, surface diffusivities for mercury was estimated by equating the surface diffusion flux to the pore diffusion flux. Dobrzelewski (1984) observed that the surface diffusivity flux was approximately a constant factor times the pore diffusivity flux for a number of organic compounds. Surface diffusivity was calculated by multiplying the pore diffusion flux by a correction factor. The correction factor was determined to have an average valve of 8 .

$$
D_{s}=\left(\frac{D_{L} \varepsilon_{p} C_{0}}{\tau_{p} \rho_{a} K C_{0}^{1 / n}}\right) * \text { Correction Factor }
$$

in which: $\quad D_{s} \quad$ surface diffusivity, $\mathrm{m}^{2} / \mathrm{s}$

$D_{L} \quad$ liquid diffusivity, $\mathrm{m}^{2} / \mathrm{s}$

$\varepsilon_{\mathrm{p}} \quad$ bed porosity

$\tau_{\mathrm{p}} \quad$ sorbent tortuosity 
$\rho_{a} \quad$ apparent density of the sorbent, $\mathrm{kg} / \mathrm{m}^{3}$

$K \quad$ Freundlich capacity constant

$C_{0} \quad$ inlet solute concentration, kgmoles $/ \mathrm{m}^{3}$

$1 / n \quad$ Freundlich intensity constant

Values for the Freundlich capacity constant, K, and the intensity constant, 1/n, for mercuric chloride on carbon were determined to be to $173,000(\mathrm{ng} / \mathrm{kg} \text { )(liters } / \mathrm{ng})^{0.85}$ and 0.85 , repectively as discussed in the previous section. The tortuosity is a measure of the actual length an adsorbing molecule must travel as compared to the radial distance travelled. Since data on this parameter was unavailable from the sorbent vendor, a value of 1 was assumed for this calculation. For a mercuric chloride concentration of $1000 \mathrm{ng} /$ liter, the surface diffusivity was calculated to be $2.2 \mathrm{x}$ $10^{-13} \mathrm{~m}^{2} / \mathrm{s}$.

The mass transfer coefficient associated with the surface diffusion mechanism is related to the surface duffusivity through the equation $k_{s}=\frac{10 D_{s}}{d_{p}(1-\varepsilon)}$ [Perry (1973)]. Applying the diameter and porosity of the sorbent to the equation, the solid phase mass transfer coefficient was found to be $1.3 \times 10^{-9} \mathrm{~m} / \mathrm{s}$.

Again, the breakthrough curve for a 2 foot adsorption column was determined by integrating equation (6) from zero to $\zeta$ for different values of $\tau$. The value of the bed length parameter $(\zeta$ ) used in the integration was 19.9 as calculated from equation (8). Estimated time for the 10\%, $50 \%$, and $90 \%$ breakthrough was 120, 191, and 277 days, respectively.

\section{The Rosen Model}

In 1952, Rosen modeled the breakthrough curve for the case in which both film resistance and intraparticle diffusion are rate controlling. The Rosen model is restricted to a bed of spherical particles and assumes a linear isotherm to describe the equilibrium between the fluid and solid phases. In the development of the model, axial dispersion was assumed to be negligible. The mass balance equation for this model becomes:

$$
\frac{-\varepsilon U_{z}}{\rho_{B}}\left(\frac{\partial C_{A}}{\partial Z}\right)_{t}=\left(\frac{\partial q_{A}}{\partial t}\right)_{z}
$$

This equation was then simplified to obtain equation (18) by writing it in terms of the dimensionless variables $X$ and $\theta$.

$$
\frac{\partial q_{A}}{\partial \theta}+\frac{\partial C_{A}}{\partial X}=0
$$

with boundary conditions:

$$
\begin{aligned}
& \mathrm{C}_{\mathrm{A}}=\mathrm{C}_{\mathrm{A} 0} \text { at } X=0 \text {; for all } \theta \\
& \mathrm{q}_{\mathrm{A}}=0 \text { at } \theta=0 \text {; for all } X
\end{aligned}
$$




$$
\begin{gathered}
X=\frac{Z \rho_{B}}{\varepsilon U_{Z}} \\
\theta=\left(t-\frac{Z}{U_{Z}}\right)
\end{gathered}
$$

The external mass transfer through the stagnant film surrounding the sorbent is given by:

$$
\left(\frac{\partial q_{A}}{\partial t}\right)=\frac{k_{f} a}{\rho_{B}}\left(C_{A}-C_{A i}\right)
$$

Where: $\quad \mathrm{C}_{\mathrm{Ai}} \quad$ concentration of component $\mathrm{A}$ at the interface of the stagnant film

$\mathrm{C}_{\mathrm{A}}$ bulk concentration of component $\mathrm{A}$ in the fluid

$k_{f} \quad$ fluid phase mass transfer coefficient, $\mathrm{m} / \mathrm{s}$

$a \quad$ interfacial area of bed per volume of bed, $\mathrm{m}^{2} / \mathrm{m}^{3}$

$\rho_{B} \quad$ bulk density of the sorbent, $\mathrm{kg} / \mathrm{m}^{3}$

The material balance for the uptake of solute by diffusion into the sphere is given by the unsteady state equation:

$$
\frac{\partial q_{i}}{\partial \theta}=\frac{D}{r^{2}} \frac{\partial}{\partial r}\left(r^{2} \frac{\partial q_{i}}{\partial r}\right)
$$

where the initial condition and boundary conditions are:

$$
\begin{aligned}
& \mathrm{q}_{\mathrm{i}}=0 \text { at } \theta=0 \text { all } \mathrm{x}, \mathrm{r} \\
& \frac{\partial q_{i}}{\partial r}=0 \text { at } \mathrm{r}=0 \text { all } X, \theta \\
& q_{i}=q_{s}=K_{D} C_{i}(X, \theta) \text { at } \mathrm{r}=\mathrm{R}
\end{aligned}
$$

Rosen solved the above system of equations for a packed bed initially free of adsorbate to give effluent concentrations curves as a function of time and bed length. The solution is expressed in equation (23):

$$
\frac{C_{A}}{C_{A o}}=\frac{1}{2}+\psi(v, X, Y)
$$


Where: $v=\frac{D_{A} K_{D}}{R k_{f}} ; \quad X=\frac{3 D_{A} K_{D} Z}{m U_{Z} R^{2}} ; \quad Y=\frac{2 D_{A}}{R^{2}}\left(t-\frac{Z}{U_{Z}}\right) ; \quad m=\frac{\varepsilon}{(1-\varepsilon)}$

For values of $\mathrm{X}$ greater than 40, equation (23) has the analytical solution shown below.

$$
\frac{C_{A}}{C_{A o}}=\frac{1}{2}\left(1+\operatorname{erf}\left(\frac{\frac{3 Y}{2 X}-1}{2 \sqrt{\frac{v}{X}}}\right)\right)
$$

Values of the parameters used in the calculation of the breakthrough curve are listed below:

$\begin{array}{ll}\mathrm{K}_{\mathrm{D}} & 63,000 \mathrm{~L} / \mathrm{kg} \\ \rho_{\mathrm{L}} & 1.0 \mathrm{~kg} / \mathrm{L} \\ \mathrm{R} & 1.6 \times 10^{-3} \mathrm{~m} \\ \mathrm{k}_{\mathrm{f}} & 1.5 \times 10^{-5} \mathrm{~m} / \mathrm{s} \\ \mathrm{m} & \varepsilon /(1-\varepsilon)=0.88 \\ \mathrm{U}_{\mathrm{Z}} & 2.2 \times 10^{-3} \mathrm{~m} / \mathrm{s} \\ \mathrm{D}_{\mathrm{A}} & 2.0 \times 10^{-13} \mathrm{~m}^{2} / \mathrm{s}\end{array}$

The breakthrough curve for a 2 foot adsorption column was calculated from equation (24). Estimated times for the 10\%, 50\%, and 90\% breakthrough are 126 days, 246 days, and 366 days, respectively.

\section{Comparison of Model Results}

Breakthrough curves predicted by the three models for the uptake of mercury from water are shown in Figure 27. As expected the Rosen model predicted longer breakthrough times since it describes the mass transfer rate for both fluid and intraparticle diffusion. 


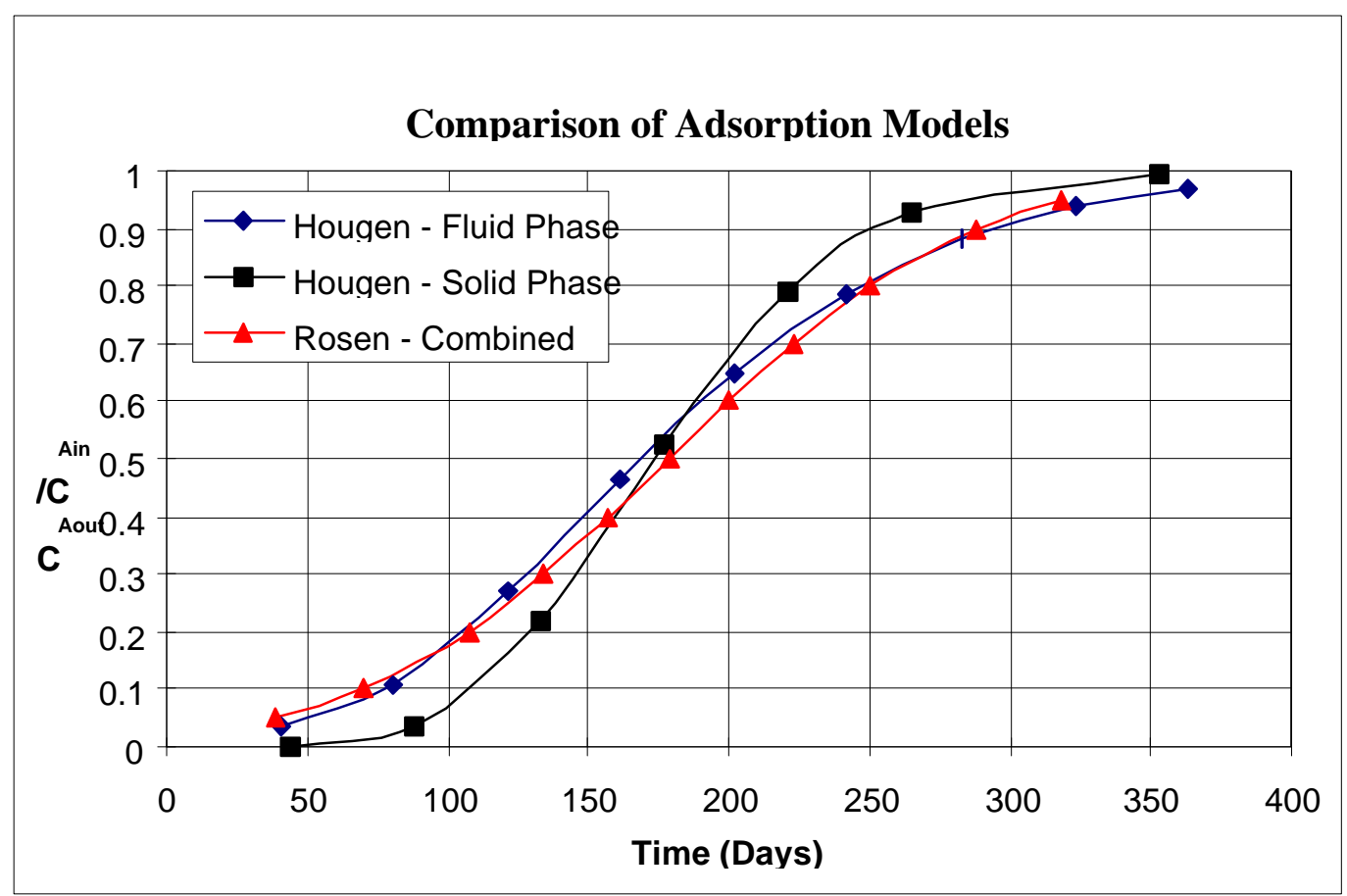

Figure 27 -- Comparison of Sorption Models 